\title{
A NUMERICAL CRITERION FOR GENERALISED MONGE-AMPÈRE EQUATIONS ON PROJECTIVE MANIFOLDS
}

\author{
Ved V. Datar and Vamsi Pritham Pingali
}

\begin{abstract}
We prove that generalised Monge-Ampère equations (a family of equations which includes the inverse Hessian equations like the $J$-equation, as well as the Monge-Ampère equation) on projective manifolds have smooth solutions if certain intersection numbers are positive. As corollaries of our work, we improve a result of Chen (albeit in the projective case) on the existence of solutions to the $J$-equation, and prove a conjecture of Székelyhidi in the projective case on the solvability of certain inverse Hessian equations. The key new ingredient in improving Chen's result is a degenerate concentration of mass result. We also prove an equivariant version of our results, albeit under the assumption of uniform positivity. In particular, we can recover existing results on manifolds with large symmetry such as projective toric manifolds.
\end{abstract}

\section{Introduction}

The Monge-Ampère equation and related PDE such as the J-equation [DON99, CHEN00, SW08, WEI06, WEI04, CS17, LS15, FLM11, FLSW14, XIA16, XIAO16, DK19, YAO15, HJ17, SW] and the deformed Hermitian Yang-Mills (dHYM) equation [JY17, CJY20, CXY18, JAC, YAM18, PIN19, HJ21, HY, PIN19, CHEN21, SS19, HJ, TAK20, TAK21, CLT, KY20] play an important role in Kähler geometry. Very often, their solvability hinges on the existence of a subsolution (which, in a sense, functions as a "barrier"). Unlike the Monge-Ampère equation, which can be solved in every Kähler class [YAU78], other equations have complicated necessary conditions that are almost as hard to verify as solving the equations themselves. To remedy this problem, numerical criteria akin to the Nakai-Moizeshon criterion for projective manifolds (and the Demailly-Paun [DP01] criterion in the Kähler case) have been conjectured [SZE18, CJY20] to be sufficient for the existence of smooth solutions. Results supporting such conjectures had been proven earlier under some symmetry assumptions (i.e., the toric case) [CS17, PIN19], but recently, building on the work of Demailly and Paun [DP01], Chen [CHEN21] proved far more general results in the case of the J-equation and the dHYM equation. The aim of this paper is to prove similar results on projective manifolds for generalised Monge-Ampère equations [PIN14] - a family of PDE which includes the Monge-Ampère equations, the inverse Hessian equations, and special cases of the dHYM equations. 
The setting is as follows. Let $(M, \chi)$ be a projective Kähler manifold and let $\left[\Omega_{0}\right]$ be a Kähler class on $M$. Let $c_{k} \geq 0,1 \leq k \leq n-1$ be constants and $f$ be a smooth function such that either

$$
\sum_{k=1}^{n-1} c_{k}=0, f>0
$$

or

$$
\sum_{k=1}^{n-1} c_{k}>0, \quad \int_{M} f \chi^{n} \geq 0 .
$$

Assume that the following condition is met.

$$
\int_{M}\left[\Omega_{0}\right]^{n}=\sum_{k=1}^{n-1} \int_{M} c_{k}[\chi]^{n-k}\left[\Omega_{0}\right]^{k}+\int_{M} f \chi^{n} .
$$

Here, and in what follows, we will suppress the wedge product to condense notation. That is, if $\alpha$ and $\beta$ are two forms or cohomology classes, then we denote $\alpha \wedge \beta$ by $\alpha \beta$. Furthermore, we assume that

$f>f_{m}=-\min \left(\frac{1}{16 n}\left(\frac{\zeta c_{\zeta}}{2 n}\right)^{\frac{\zeta}{n-\zeta}} \frac{c_{\zeta}(n-\zeta)}{2 n}, \frac{\zeta c_{\zeta}^{2}}{4 n}, \frac{c_{\zeta}^{\frac{n}{n-\zeta}}}{4 n}, \frac{\int_{M}\left[\Omega_{0}\right]^{n}}{4 \int_{M}[\chi]^{n}}, \frac{K}{2 n}\left(\frac{c_{\zeta}}{2\left(\begin{array}{c}n \\ \zeta\end{array}\right)}\right)^{\frac{n}{n-\zeta}}\right)$,

where $1 \leq \zeta \leq n-1$ is the largest integer such that $c_{\zeta} \neq 0$, and $1>K>0$ is any constant less than the smallest eigenvalue of the matrix $\sum_{|I|=\zeta} E_{I}$ where $I$ is a multi-index and $\left(E_{I}\right)_{i j}=1$ if $i, j \notin I$ and 0 otherwise. Our main result is the following.

Theorem 1.1. Let $M$ be a projective connected $n$-dimensional manifold. Let $\chi$ be a fixed Kähler metric, and $\left[\Omega_{0}\right]$ be a Kähler class. Furthermore, let $f: M \rightarrow \mathbb{R}$ be a smooth function and $\left\{c_{k}\right\}_{k=1}^{n-1}$ constants satisfying the conditions above. Then the following conditions are equivalent.

(1) The generalised Monge-Ampère equation

$$
\Omega^{n}=\sum_{k=1}^{n-1} c_{k} \chi^{n-k} \Omega^{k}+f \chi^{n},
$$

has a unique smooth solution $\Omega \in\left[\Omega_{0}\right]$ satisfying the cone condition

$$
n \Omega^{n-1}-\sum_{k=1}^{n-1} c_{k} k \chi^{n-k} \Omega^{k-1}>0
$$


(2) (Cone condition) There exists a Kähler metric $\Omega \in\left[\Omega_{0}\right]$ satisfying the cone condition, i.e.,

$$
n \Omega^{n-1}-\sum_{k=1}^{n-1} c_{k} k \chi^{n-k} \Omega^{k-1}>0 .
$$

(3) (Numerical condition) For all subvarieties $V \subset M$ of co-dimension $p$, we have

$$
\int_{V}\left(\left(\begin{array}{l}
n \\
p
\end{array}\right)\left[\Omega_{0}\right]^{n-p}-\sum_{k=p}^{n-1} c_{k}\left(\begin{array}{l}
k \\
p
\end{array}\right)\left[\chi^{n-k}\right] \cdot\left[\Omega_{0}^{k-p}\right]\right)>0 .
$$

Note that even for the $J$-equation, this is an improvement of Gao Chen's result [CHEN21, Theorem 1.1], at least for projective manifolds. Theorem 1.1 immediately implies Conjecture 23 of Székelyhidi [SZE18] (which itself generalises a conjecture of Lejmi-Székelyhidi [LS15]) for the inverse Hessian equations in the projective case.

Corollary 1.2. Let $\left[\Omega_{0}\right]$ be a Kähler class and $\chi$ be a Kähler form on a projective manifold $M$. Define the constant $c:=\frac{\int_{M}\left[\Omega_{0}\right]^{n}}{\int_{M}\left[\Omega_{0}\right]^{n-k}[\chi]^{k}}$. The inverse Hessian equation

$$
\Omega^{n}=c \Omega^{n-k} \wedge \chi^{k}
$$

has a solution if and only if for every subvariety $V$ of codimension $n-k \leq p \leq n-1$, the following inequality holds.

$$
\int_{V}\left(\begin{array}{l}
n \\
p
\end{array}\right)\left[\Omega_{0}\right]^{n-p}-\int_{V} c\left(\begin{array}{l}
k \\
p
\end{array}\right)[\chi]^{k}\left[\Omega_{0}\right]^{n-p-k}>0 .
$$

To prove Theorem 1.1, it is first necessary to obtain a version of the theorem assuming uniform positivity of intersection numbers. Namely, we first prove the following, which is an exact extension of [CHEN21, Theorem 1.1] to more general inverse Hessian equations on projective manifolds. In fact, we can prove an equivariant version.

Theorem 1.3. Assume that a connected compact Lie group $G$ acts on $M$ by biholomorphisms such that $M$ is strongly $G$-compatible, and $G$ preserves $\chi$ and $f$. The following conditions are equivalent.

(1) The generalised Monge-Ampère equation

$$
\Omega^{n}=\sum_{k=1}^{n-1} c_{k} \chi^{n-k} \Omega^{k}+f \chi^{n}
$$

has a unique smooth $G$-invariant solution $\Omega \in\left[\Omega_{0}\right]$ satisfying the cone condition

$$
n \Omega^{n-1}-\sum_{k=1}^{n-1} c_{k} k \chi^{n-k} \Omega^{k-1}>0 .
$$


(2) (Cone condition) There exists a $G$-invariant Kähler metric $\Omega \in\left[\Omega_{0}\right]$ satisfying the cone condition, i.e.,

$$
n \Omega^{n-1}-\sum_{k=1}^{n-1} c_{k} k \chi^{n-k} \Omega^{k-1}>0 .
$$

(3) (Uniform numerical condition) There exists a constant $\varepsilon>0$ such that for all $G$-invariant subvarieties $V \subset M$ of co-dimension $p$, we have

$$
\int_{V}\left(\left(\begin{array}{l}
n \\
p
\end{array}\right)\left[\Omega_{0}\right]^{n-p}-\sum_{k=p}^{n-1} c_{k}\left(\begin{array}{l}
k \\
p
\end{array}\right)\left[\chi^{n-k}\right] \cdot\left[\Omega_{0}^{k-p}\right]\right) \geq \varepsilon\left(\begin{array}{l}
n \\
p
\end{array}\right) \int_{V}\left[\Omega_{0}\right]^{n-p} .
$$

Before proceeding, we explain the $G$-compatibility. We say that $M$ is $G$-compatible if every $G$-invariant closed analytic subset contains an ample $G$-invariant divisor. Furthermore, suppose $Z$ is a $G$-invariant closed analytic subset. Consider a canonical resolution of singularities (which always exists by the results of [KOL07, BM99] that improve on the foundational work of Hironaka on a possibly non-canonical resolution [HIR64, HIRO64]) $Z_{0}=Z \leftarrow Z_{1} \leftarrow \cdots \leftarrow Z_{r}$ obtained by blowing up $M$ successively (to get $M_{i}$ ) at $G$-invariant (with respect to the lifted $G$-action on $M_{i}$ ) smooth centres $C_{i} \subset Z_{i}$ and taking the proper transform. If for any $Z$ and any such canonical resolution, $M_{i}$ is $G$-compatible for all $i$, then $M$ is defined to be strongly $G$-compatible. Note that in this paper, "G-invariant divisor" simply means that the union of the irreducible subvarieties of the divisor is a $G$-invariant set. (That is, the multiplicities are not considered in this terminology.)

REMARK 1.1. A projective toric manifold $M$ is strongly $\left(S^{1}\right)^{n}$-compatible. Indeed, there is an ample toric line bundle $L$ over $M$ that restricts to an ample toric line bundle over every toric subvariety $Z$. Hence, for large $k$, taking the zero locus of a section of $L^{k}$ that generates an invariant subspace (for the lifted action of $\left(S^{1}\right)^{n}$ ), we get an ample invariant divisor. Blowing up a projective toric variety at a smooth toric subvariety gives a toric variety with a toric ample line bundle $L^{k} \otimes[-E]$ for sufficiently large $k$. The same argument as above shows that smooth projective $T$-varieties, i.e., projective manifolds with an effective $G=\left(S^{1}\right)^{k}$ action (where $0 \leq k \leq n)$, are also strongly $G$-compatible.

REMARK 1.2. If $c_{k}=0 \forall k$ and $f>0$ everywhere, then the equation (1.4) reduces to the usual Monge-Ampere equation, and hence Theorem 1.3 follows from the proof of Calabi conjecture by [YAU78], and the numerical characterization of the Kähler cone by Demailly and Paun [DP01]. The statement $1 \Leftrightarrow 2$ is known in the case $f \geq 0$ in much greater generality. (However, very few cases where $f$ is allowed to be negative appear to be studied [CHEN21, ZHE15, PIN19].) The case of the J-equation was studied originally in [CHEN00, SW08, WEI06]. Other inverse Hessian-type equations were first studied in [FLM11].

We recover many other existing results when they are specialised to the projective case. If $G$ is taken to be trivial, $c_{k}=0$ for $k=1,2 \cdots, n-2$, and $c_{n-1}>0$, then 
this result reduces to the main theorem in [CHEN21]. If $G=\left(S^{1}\right)^{n}$, we recover the main theorem in [CS17]. Note that in the toric case, $G$-equivariant positivity of the numerical condition and its uniform version are equivalent. More generally, by Remark 1.1, our theorem also applies to $T$-varieties. For certain values of the coefficients and phase angles, as explained in [PIN19], the dHYM equation is a generalised Monge-Ampère equation with non-negative coefficients. Hence, for such phase angles we recover the results of [CHEN21] when $G$ is trivial and those of [PIN19] when $G=\left(S^{1}\right)^{n}$. As far as we know, our results on generalised MongeAmpère equations on projective manifolds are new in the case of $G=\left(S^{1}\right)^{k}$ (where $1 \leq k \leq n-1)$.

REMARK 1.3. (A comparison with Chen's work [CHEN21]) The argument of Chen in [CHEN21] relies on the "diagonal trick" of Demailly and Paun. This involves solving a certain $J$-equation on the product $M \times M$ and obtaining currents that concentrate along the diagonal $\Delta \subset M \times M$. Though our strategy of proof is inspired from [CHEN21], replicating the diagonal trick in the setting of more general inverse Hessian equations poses significant additional technical challenges. In particular, it is not clear what equation must one solve on $M \times M$. The extension of our theorem to all Kähler manifolds is a work in progress. In this paper, we instead take a different route and use divisors as barriers, and rely on the existence of many sub-varieties on projective manifolds. More crucially we are able to improve Chen's result, even in the case of the $J$ equation (albeit only on projective manifolds), in that we assume only positivity of the intersection numbers, as opposed to uniform positivity in [CHEN21, Theorem 1.1]. This is one of the main contributions of the present work.

REMARK 1.4 (A comparison to the recent preprint of Jian Song [SON]). In [SON], Jian Song proved a version of Theorem 1.1 for the $J$-equation on Kähler manifolds using a method that parallel's Gao Chen's method in [CHEN21]. Our attempts at relaxing the uniform numerical criteria in Theorem 1.3 grew, in part, out of some discussions with him. Note that the key ingredient in both our work and Song's work is a degenerate mass concentration result (compare Proposition 5.2 to [SON, Theorem 5.1]). It would be interesting to remove the projectivity assumption in Theorems 1.3 and 1.1, but as remarked above, there are new technical difficulties that need to be overcome.

REMARK 1.5. Using a reformulation of the dHYM equation as a generalised MongeAmpère equation, Theorem 1.1 implies that in the three-dimensional case, when $\tan (\hat{\theta}) \geq 0$, positivity of some intersection numbers is equivalent to existence of a solution. The result in [CHEN21] proved such an assertion only under the uniform positivity assumption. Recently, $[\mathrm{CLT}]$ proved the the most general result in this direction.

Remark 1.6. Combining Theorem 1.1 above with Theorem 5 and Remark 1 in [DYR20], it follows that the optimal lower bound for the $J$-functional is attained by some sub-variety. We refer the interested reader to [DYR20] for more details. 
We end this section with outlines of the proofs of Theorem 1.3 and Theorem 1.1. As mentioned earlier, the proof of Theorem 1.1 relies on the weaker Theorem 1.3.

Outline of the proof of Theorem 1.3. We only need to focus on the implication $3 \Rightarrow 1$ because the others are somewhat standard. In fact since (1) Theorem 2.1 , we actually prove that $(3) \Longrightarrow(2)$. That is, given the numerical condition, we produce a $G$-invariant $\Omega \in\left[\Omega_{0}\right]$ satisfying the cone condition. Just as in [DP01, CHEN21], we proceed by means of a continuity method. For $t \geq 0$, consider the equation

$$
\Omega_{t}^{n}=\sum_{k=1}^{n-1} c_{k} \chi^{n-k} \Omega_{t}^{k}+f \chi^{n}+a_{t} \chi^{n}
$$

where $\Omega_{t} \in(1+t)\left[\Omega_{0}\right]$. Note that $a_{t} \stackrel{t \rightarrow 0^{+}}{\longrightarrow} 0$, and hence we want to solve the above equation at $t=0$. For $t>>1, \hat{\Omega}_{t}=(1+t) \Omega_{0}$ satisfies the cone condition

$$
n \hat{\Omega}_{t}^{n-1}-\sum_{k=1}^{n-1} c_{k} k \chi^{n-k} \hat{\Omega}_{t}^{k-1}>0 .
$$

Moreover, for all $t \geq 0$, the assumptions on $f_{m}$ are met. By Theorem 2.1, there exists a solution to (4.11) for $t>>1$. In particular, if we let

$$
I=\{t \in[0, \infty) \mid(1.5) \text { has a solution }\}
$$

then $I$ is non-empty. The infinite-dimensional implicit function theorem implies that $I$ is open. We need to show that the set is closed. By the nature of the cone condition, if $t \in I$, then $t^{\prime} \in I$ for all $t^{\prime}>t$. So let $t_{0}=\inf I$. It is enough to prove that $t_{0} \in I$. Replacing $\Omega_{0}$ by $\left(1+t_{0}\right) \Omega_{0}$, without loss of generality, we may assume that $t_{0}=0$ i.e. we have a solution to (4.11) for all $t>0$.

- Step-1. Let $Y$ be a $G$-invariant ample divisor (which exists by hypothesis). By Theorem 3.1, there is a closed, non-negative current $\Theta \in\left[\Omega_{0}\right]$ such that $\Theta \geq 2 \beta[Y]$ for some $\beta<<1$, and $\Theta-\beta[Y]$ satisfies the cone condition (not necessarily with strict positivity though) outside $Y$ in the sense of Chen.

- Step-2. For any $c>0$, the Lelong sub-level set $E_{c}=\{x \mid \nu(\Theta-\beta[Y], x) \geq c\}$ is a closed analytic subset that is $G$-invariant if $\Theta$ and $Y$ are $G$-invariant. Let $Z=E_{c} \cup Y$, and let $Z \subset V \subset \subset U$. In a neighbourhood $U$ of $Z$, we construct a metric $\Omega_{U}=\Omega_{0}+\sqrt{-1} \partial \bar{\partial} \psi_{U}$ satisfying the cone condition using the induction hypothesis and the existence of $G$-equivariant resolutions of singularities (which follows from functoriality in [BM99, KOL07]) (cf. Lemma 4.4).

- Step-3. Let $\chi_{Y} \in[Y]$ be a Kähler metric. Outside a neighbourhood (of predetermined size) $U$ of the $G$-invariant ample divisor $Y$, we regularise $T=$ $\Theta-\beta[Y]+\beta \chi_{Y}$ to obtain a metric in $\left[\Omega_{0}\right]$ satisfying the cone condition on $U^{c}$. 
- Step-4. Using Proposition 4.1 we glue the metrics obtained in Step-2 and Step3 to get a smooth metric satisfying the cone condition. In the $G$-equivariant case, the metric obtained can be averaged over $G$ to make it $G$-invariant. Since $G$ is connected and compact, the averaging process does not change the Kähler class. Moreover, since the cone condition can be written in a convex manner, the averaged metric continues to satisfy it, and we are done.

Outline of the proof of Theorem 1.1. The proof of Theorem 1.3 carries through almost verbatim, as long as one can construct a Kähler metric satisfying the (nonuniform) cone condition in the neighbourhood of any analytic subset $Z \subset M$ (cf. Proposition 5.1). If $Z$ is smooth, this again follows almost verbatim from the proof of Lemma 4.3. In general we use a resolution of singularities, a stronger mass concentration lemma for certain semi-ample classes (cf. Proposition 5.2), and an induction on the maximal dimension of an irreducible component of $Z$. During the course of our proof, we need to choose an ample divisor $Y$ (akin to Step-1 in the proof of Theorem 1.3) satisfying some properties. It is not clear to us if such a choice can be made in the $G$-invariant case (even under the assumption of $G$-compatibility).

\section{The PDE Aspects}

In this section we prove that the generalised Monge-Ampère equation with a slight negative "constant" term can be solved (if $\sum_{k} c_{k}>0$ ) assuming the cone condition holds.

Theorem 2.1. $\quad(1) \Leftrightarrow(2)$ in Theorem 1.3.

We prove Theorem 2.1 using the method of continuity. Uniqueness is standard. Given uniqueness, the equivariant version of the theorem follows easily. Hence, we may ignore $G$ for the remainder of this section. Since the case $c_{k}=0 \forall k, f>0$ is well-known [YAU78], we assume without loss of generality that $\sum_{k} c_{k}>0$.

Note that since $\int_{M} f \chi^{n} \geq 0$, there is a point $p \in M$ where $f(p) \geq 0$. At such a point, it is easy to see that the cone condition is met. This observation combined with the following lemma shows the necessity of the cone condition at all points on $M$.

Lemma 2.1. Suppose $f(p) \geq 0$ for some $p \in M$. Assume that Equation 1.4 has a smooth solution $\Omega$. Then $\Omega>0$ and $n \Omega^{n-1}-\sum_{k=1}^{n-1} c_{k} \chi^{n-k} k \Omega^{k-1}>0$.

Proof. Let $q \in M$ be any other point and $\gamma(s)$ be a path connecting $p$ to $q$. Assume that $r$ is the first point on $\gamma(s)$ where either the cone condition or the Kähler condition becomes degenerate. It is easy to see that the cone condition must become degenerate first. We will rule out this possibility, hence showing that neither can become degenerate. 
If $f(r) \geq 0$, the result is standard. Therefore, without loss of generality assume that $f(r)<0$. Choose holomorphic normal coordinates for $\chi$ at $r$ such that $\Omega$ is diagonal with eigenvalues $\lambda_{1} \leq \lambda_{2} \ldots$. Equation 1.4 can be rewritten as

$$
1=\sum_{k=1}^{n-1} c_{k} \frac{k !(n-k) !}{n !} S_{n-k}\left(\frac{1}{\lambda}\right)+f S_{n}\left(\frac{1}{\lambda}\right),
$$

where the symmetric polynomial $S_{k}(A)$ is the coefficient of $t^{k}$ in $\operatorname{det}(I+t A)$. The cone condition can be written as

$$
1>\sum_{k=1}^{n-1} c_{k} \frac{k !(n-k) !}{n !} S_{n-k ; i}\left(\frac{1}{\lambda}\right),
$$

where $S_{n-k ; i}\left(\frac{1}{\lambda}\right)$ is a symmetric polynomial in $n-1$ eigenvalues (excluding $\lambda_{i}$ ). For future use, we define $S_{n-k ; i, j}=0$ when $i=j$ and the symmetric polynomial in $n-2$ eigenvalues (excluding $\lambda_{i}, \lambda_{j}$ ) when $i \neq j$. When it degenerates, for some $i$,

$$
1=\sum_{k=1}^{n-1} c_{k} \frac{k !(n-k) !}{n !} S_{n-k ; i}\left(\frac{1}{\lambda}\right) .
$$

Denote $S_{k}\left(\frac{1}{\lambda}\right)$ by $\sigma_{k}$. Using $\sigma_{n-k}=\sigma_{n-k ; i}+\frac{\sigma_{n-k-1 ; i}}{\lambda_{i}}$ we arrive at the following equations.

$$
\begin{aligned}
0 & =\sum_{k=1}^{n-1} c_{k} \frac{k !(n-k) !}{n ! \lambda_{i}} \sigma_{n-k-1 ; i}+f \sigma_{n} \\
\Rightarrow \sigma_{n-1 ; i} & =\frac{1}{|f|} \sum_{k=1}^{n-1} c_{k} \frac{k !(n-k) !}{n !} \sigma_{n-k-1 ; i} \geq \frac{c_{\zeta} \sigma_{n-\zeta-1 ; i}}{\left(\begin{array}{l}
n \\
\zeta
\end{array}\right)|f|} .
\end{aligned}
$$

At this juncture, we use the Maclaurin inequality to simplify the expression in 2.4.

$$
\begin{gathered}
\frac{c_{\zeta} \sigma_{n-\zeta-1 ; i}}{\left(\begin{array}{c}
n \\
\zeta
\end{array}\right)|f|} \geq \frac{c_{\zeta}\left(\begin{array}{c}
n-1 \\
\zeta
\end{array}\right) \sigma_{n-1 ; i}^{\frac{n-\zeta-1}{n-1}}}{\left(\begin{array}{c}
n \\
\zeta
\end{array}\right)|f|} \\
\Rightarrow \sigma_{n-1 ; i} \geq\left(\frac{c_{\zeta}(n-\zeta)}{n|f|}\right)^{\frac{n-1}{\zeta}} .
\end{gathered}
$$

From 2.3 we see that

$$
\begin{gathered}
1 \geq \frac{c_{\zeta}}{\left(\begin{array}{c}
n \\
\zeta
\end{array}\right)} \sigma_{n-\zeta ; i} \\
\Rightarrow \frac{\left(\begin{array}{c}
n \\
\zeta
\end{array}\right)}{c_{\zeta}} \geq \sigma_{n-\zeta ; i} \geq\left(\begin{array}{c}
n-1 \\
\zeta-1
\end{array}\right) \sigma_{n-1 ; i}^{\frac{n-\zeta}{n-1}}
\end{gathered}
$$


Comparing 2.6 and 2.5 we see that

$$
|f| \geq\left(\frac{\zeta c_{\zeta}}{n}\right)^{\frac{\zeta}{n-\zeta}} \frac{c_{\zeta}(n-\zeta)}{n} .
$$

By the assumption on $f$ we arrive at a contradiction. Hence the cone condition never degenerates. From the cone condition, we can clearly see that Kählerness also holds.

Now we prove existence assuming that the background metric $\hat{\Omega}_{0} \in\left[\Omega_{0}\right]$ satisfies the cone condition. To this end, consider the following continuity path depending on a parameter $0 \leq t \leq 1$.

$$
\Omega_{t}^{n}=t\left(\sum_{k=1}^{n-1} c_{k} \chi^{n-k} \Omega_{t}^{k}+f \chi^{n}\right)+(1-t) c_{0} \chi^{n},
$$

where $c_{0}$ is a constant such that $c_{0} \int_{M}[\chi]^{n}=\int_{M}\left[\Omega_{0}\right]^{n}$. For a smooth function $\phi$, let $\Omega_{\phi}=\hat{\Omega}_{0}+\sqrt{-1} \partial \bar{\partial} \phi$. We normalise $\phi$ to satisfy inf $\phi=0$. At $t$, denote by $\phi_{t}$ the unique such smooth function so that $\Omega_{t}=\hat{\Omega}_{0}+\sqrt{-1} \partial \bar{\partial} \phi_{t}$. At $t=0$, there exists a smooth solution thanks to Yau's resolution of the Calabi conjecture. For $0 \leq t \leq \frac{1}{2}$, we see that $t f \chi^{n}+(1-t) c_{0} \chi^{n}>0$ by the assumptions on $f$. For $t>\frac{1}{2}$, we see (using the assumptions on $f$ again) that $t f+(1-t) c_{0}>-\left(\frac{t \zeta c_{\zeta}}{n}\right)^{\frac{\zeta}{n-\zeta}} \frac{t c_{\zeta}(n-\zeta)}{n}$. The proof of Lemma 2.1 implies that the cone condition is met. Hence, the linearisation is invertible and the set of all such $t$ where there is a solution is open. To prove that it is closed, we need a priori estimates. Since we are given that the cone condition is preserved along the continuity path, using Yau's Moser iteration technique (akin to [SUN] for instance), one can easily prove a $C^{0}$ estimate. We now prove a Laplacian estimate.

Proposition 2.1. $\Delta_{\chi} \phi_{t} \leq C$ where $C$ is independent of $t$.

Proof. First we prove the following lemma.

Lemma 2.2. For each $0 \leq t \leq 1$, let $S_{t, p}$ be the open subset of positive-definite Hermitian matrices satisfying the cone condition for Equation 2.8. Cover $M$ with finitely many holomorphic charts. For a fixed point $p$, the function $F_{t, p}: S_{t, p} \rightarrow \mathbb{R}$ given by

$$
F_{t, p}(A)=\sum_{k=1}^{n-1} t \frac{c_{k}}{\left(\begin{array}{l}
n \\
k
\end{array}\right)} \sigma_{n-k}(A)+\left(t f(p)+(1-t) c_{0}\right) \sigma_{n}(A)
$$

satisfies the following properties. Let $A$ have eigenvalues $\lambda_{i}>0$ and let $L$ be any constant larger than the maximum over all the coordinate charts of $\left|\frac{\partial f}{\partial z^{\mu}}\right|$ and $\left|\frac{\partial^{2} f}{\partial z^{\mu} \partial \bar{z}^{\mu}}\right|$.

(1) $S_{t}$ is a convex open set. 
(2) The function $g_{t, p}(A)=F_{t, p}\left(A^{-1}\right)$ extends to a smooth function (of $t, p, A$ ) on the orthant $\left\{\lambda_{i} \geq 0\right\}$ (and $0 \leq t \leq 1, p \in M$ ).

(3) $\frac{\partial F_{t, p}}{\partial \lambda_{i}}(A)<0$.

(4) If $\lambda_{1} \leq \lambda_{2} \ldots$, then

$$
-\lambda_{1} \frac{\partial F_{t, p}}{\partial \lambda_{1}} \geq-\lambda_{i} \frac{\partial F_{t, p}}{\partial \lambda_{i}} \forall i
$$

(5) If $B$ is any Hermitian matrix, then

$$
\sum_{\mu, \nu, \alpha, \beta} \frac{\partial^{2} F_{t, p}}{\partial A_{\mu \nu} \partial A_{\alpha \beta}} \bar{B}_{\nu \mu} B_{\alpha \beta}+\sum_{i, j} \frac{\partial F_{t, p}}{\partial \lambda_{i}} \frac{\left|B_{i j}\right|^{2}}{\lambda_{j}}-\alpha \sum_{i} \frac{\partial F_{t, p}}{\partial \lambda_{i}} \frac{\left|B_{i i}\right|^{2}}{\lambda_{i}} \geq 0 .
$$

where $\alpha=0$ if $2 \zeta \geq n$ and $\alpha=\frac{1}{2}$ otherwise. In particular, for all $1 \leq \zeta \leq n-1$, we see that $F_{t, p}$ is convex.

(6) $F_{t, p}(A)>0$.

(7) There is a constant $C$ independent of $t, p, A$ such that

$$
\frac{F_{t, p}}{C} \leq \sum_{k}-\lambda_{k} \frac{\partial F_{t, p}}{\partial \lambda_{k}} \leq C F_{t, p}
$$

(8) $\sum_{\mu}\left|\frac{\partial^{2} F_{t, p}(A)}{\partial z^{\mu} \bar{z}^{\mu}}\right| \leq C$ where $C$ is independent of $t, p, A$.

Suppose that there is a matrix $A_{0} \in \cap_{0 \leq t \leq 1} S_{t}$ with eigenvalues $0<\mu_{1} \leq \mu_{2} \ldots \leq \mu_{n}$. If $F_{t}(A, p)=1$ for all $p \in M$, then

(9) There exist constants $\theta, N>0$ independent of $t, p, A$ such that if $\operatorname{tr}(A)>N$, then $\sum_{i} \frac{\partial F_{t, p}}{\partial \lambda_{i}}(A)\left(\lambda_{i}-\mu_{i}\right) \geq \theta\left(1-\sum_{i} \frac{\partial F_{t, p}}{\partial \lambda_{i}}(A)\right)$.

(10) There exists a constant $C$ independent of $t, p, A$ such that for any matrix $B$ and any number $\epsilon>0$,

$$
\sum_{i, \mu}\left|\frac{\partial^{2} F_{t, p}(A)}{\partial \lambda_{i} \partial z^{\mu}} B_{i \mu}\right| \leq \frac{C}{\epsilon}-\epsilon \sum_{i, \mu} \frac{\partial F_{t, p}(A)}{\partial \lambda_{i}} \frac{\left|B_{i \mu}\right|^{2}}{\lambda_{i}}
$$

Proof. Note that the cone condition is $1>\sum_{k=1}^{n-1} \frac{t c_{k}}{\left(\begin{array}{l}n \\ k\end{array}\right)} \sigma_{n-k ; i}$. The first few statements are proved as follows.

(1) Since $\sigma_{n-k ; i}$ is well known to be a convex function, $S_{t}$ is an open convex set.

(2) Trivial.

(3)

$$
-\frac{\partial F_{t}}{\partial \lambda_{i}}=\frac{1}{\lambda_{i}}\left(\sum_{k=1}^{n-1} \frac{t c_{k}}{\left(\begin{array}{l}
n \\
k
\end{array}\right)} \frac{1}{\lambda_{i}} \sigma_{n-k-1 ; i}+\left(t f+(1-t) c_{0}\right) \sigma_{n}\right) .
$$

When $0 \leq t \leq \frac{1}{2}$ the result is clear because the $\sigma_{n}$ term is non-negative. If $\frac{1}{2} \leq t \leq 1$, then using the Maclaurin inequality we arrive at the following inequalities. 


$$
\begin{aligned}
-\lambda_{i}^{2} \frac{\partial F_{t}}{\partial \lambda_{i}} & \geq \frac{c_{\zeta} \sigma_{n-\zeta-1 ; i}}{2\left(\begin{array}{c}
n \\
\zeta
\end{array}\right)}-\left|f_{m}\right| \sigma_{n-1 ; i} \geq \frac{c_{\zeta}\left(\begin{array}{c}
n-1 \\
n-\zeta-1
\end{array}\right)}{2\left(\begin{array}{c}
n \\
\zeta
\end{array}\right)} \sigma_{n-1 ; i}^{\left.\frac{\left(\begin{array}{c}
n-2 \\
n-\zeta-2
\end{array}\right)}{n-\zeta-1}\right)}-\left|f_{m}\right| \sigma_{n-1 ; i} \\
& =\sigma_{n-1 ; i}^{\frac{n-\zeta-1}{n-1}}\left(\frac{c_{\zeta}(n-\zeta)}{2 n}-\left|f_{m}\right| \sigma_{n-1 ; i}^{\frac{\zeta}{n-1}}\right)
\end{aligned}
$$

which is seen to be positive using the cone condition and the assumptions on $f_{m}$.

(4) Note that for $k<n,-\lambda_{1} \partial_{1} \sigma_{k} \geq-\lambda_{i} \partial_{i} \sigma_{k}$ and for $k=n,-\lambda_{1} \partial_{1} \sigma_{n}=-\lambda_{i} \partial_{i} \sigma_{n}$. Hence, the result follows.

(5) From the proof of Lemma 8 in [CS17], we see that

$$
\begin{aligned}
& \sum_{\mu, \nu, \alpha, \beta} \frac{\partial^{2} F_{t, p}}{\partial A_{\mu \nu} \partial A_{\alpha \beta}} \bar{B}_{\nu \mu} B_{\alpha \beta}+\sum_{i, j} \frac{\partial F_{t, p}}{\partial \lambda_{i}} \frac{\left|B_{i j}\right|^{2}}{\lambda_{j}} \\
& \quad \geq \sum \frac{t c_{k}}{\left(\begin{array}{l}
n \\
k
\end{array}\right)} \frac{B_{\alpha \alpha} \bar{B}_{\mu \mu}}{\lambda_{\alpha} \lambda_{\mu}} \frac{S_{k ; \alpha, \mu}+\delta_{\alpha \mu} S_{k ; \alpha}}{S_{n}}+\frac{t f+(1-t) c_{0}}{S_{n}} \frac{B_{\alpha \alpha} \bar{B}_{\mu \mu}}{\lambda_{\alpha} \lambda_{\mu}} .
\end{aligned}
$$

The matrix $M_{i j}=\sum \frac{t c_{k}}{\left(\begin{array}{c}n \\ k\end{array}\right)}\left(S_{k ; i j}+\delta_{i j} S_{k ; i}\right)+\left(t f+(1-t) c_{0}\right)$ can be written as (akin to [FLM11, CS17]) $M=\sum \frac{t c_{k}}{\left(\begin{array}{c}n \\ k\end{array}\right)} \sum_{\|I\|=k} \lambda_{I} E_{I}+\left(t f+(1-t) c_{0}\right) \mathbf{1}$ where for any $k$-tuple $I$, the positive semidefinite matrix $E_{I}$ is defined to have entries $\left(E_{I}\right)_{i j}=1$ if $i, j \notin I$ and 0 otherwise; moreover, $\lambda_{I}=\lambda_{i_{1}} \lambda_{i_{2}} \ldots \lambda_{i_{k}}$, and the matrix $\mathbf{1}$ is defined as $(\mathbf{1})_{i j}=1 \forall i, j$.

If $0 \leq t \leq \frac{1}{2}$, it is easy to see by the assumptions on $f$ that $M$ is non-negative (because the constant term is so). Assume $t>\frac{1}{2}$. For future use, we observe that $0 \leq \mathbf{1} \leq n I d$. Now we have two cases.

(a) $2 \zeta \geq n$ :Using the cone condition and the assumption that $2 \zeta \geq n$, we see that $\lambda_{I}>\left(\frac{c_{\zeta}}{2\left(\begin{array}{c}n \\ \zeta\end{array}\right)}\right)^{\frac{\zeta}{n-\zeta}} \forall$ multi-indices $I$ with $|I|=\zeta$. Recall that $K>0$ is the least eigenvalue of $\sum_{\|I\|=\zeta} E_{I}$. Now note that $M>t\left(\frac{c_{\zeta}}{2\left(\begin{array}{l}n \\ \zeta\end{array}\right)}\right)^{\frac{n}{n-\zeta}} K I-$ $t\left|f_{m}\right| n I$, which is positive-definite using the assumptions on $f_{m}$.

(b) $1 \leq \zeta \leq n-1$ : Note that

$$
\begin{aligned}
& \sum_{\mu, \nu, \alpha, \beta} \frac{\partial^{2} F_{t, p}}{\partial A_{\mu \nu} \partial A_{\alpha \beta}} \bar{B}_{\nu \mu} B_{\alpha \beta}+\sum_{i, j} \frac{\partial F_{t, p}}{\partial \lambda_{i}} \frac{\left|B_{i j}\right|^{2}}{\lambda_{j}}-\frac{1}{2} \sum_{i} \frac{\partial F_{t, p}}{\partial \lambda_{i}} \frac{\left|B_{i i}\right|^{2}}{\lambda_{i}} \\
& \geq \sum_{i, j} \sigma_{n} M_{i j} \frac{B_{i i} B_{j j}}{\lambda_{i} \lambda_{j}}-\frac{1}{2} \sum_{i} \frac{\partial F_{t, p}}{\partial \lambda_{i}} \frac{\left|B_{i i}\right|^{2}}{\lambda_{i}}
\end{aligned}
$$

Using the first inequality in 2.13 we see that

$$
\sum_{\mu, \nu, \alpha, \beta} \frac{\partial^{2} F_{t, p}}{\partial A_{\mu \nu} \partial A_{\alpha \beta}} \bar{B}_{\nu \mu} B_{\alpha \beta}+\sum_{i, j} \frac{\partial F_{t, p}}{\partial \lambda_{i}} \frac{\left|B_{i j}\right|^{2}}{\lambda_{j}}-\frac{1}{2} \sum_{i} \frac{\partial F_{t, p}}{\partial \lambda_{i}} \frac{\left|B_{i i}\right|^{2}}{\lambda_{i}}
$$




$$
\geq 2\left(-\sum_{i} n \sigma_{n-1 ; i}\left|f_{m}\right| \frac{\left|B_{i i}\right|^{2}}{\lambda_{i}^{3}}+\frac{1}{8} \sum_{i} \frac{\left|B_{i i}\right|^{2}}{\lambda_{i}^{3}} \frac{c_{\zeta} \sigma_{n-\zeta-1 ; i}}{\left(\begin{array}{l}
n \\
\zeta
\end{array}\right)}\right) .
$$

As before, the Newton-Maclaurin inequalities and the assumptions on $\left|f_{m}\right|$ imply the desired result.

(6) If $0 \leq t \leq \frac{1}{2}$, it is clear (as before) that $F_{t}(A)>0$. If $t>\frac{1}{2}$, then

$$
F_{t}(A) \geq \frac{c_{\zeta}}{2} \sigma_{n}^{\frac{n-\zeta}{n}}-\left|f_{m}\right| \sigma_{n}=\sigma_{n}^{\frac{n-\zeta}{n}}\left(\frac{c_{\zeta}}{2}-\left|f_{m}\right| \sigma_{n}^{\frac{\zeta}{n}}\right) .
$$

At this point we see that the cone condition implies that $1>\frac{1}{2} \frac{c_{\zeta} \sigma_{n-\zeta ; i}}{\left(\begin{array}{l}n \\ \zeta\end{array}\right)}$ because $t>\frac{1}{2}$. Using the Maclaurin inequality we see that

$$
\begin{aligned}
& \frac{2 n}{\zeta c_{\zeta}}>\sigma_{n-1 ; i}^{\frac{n-\zeta}{n-1}} \\
& \quad \Rightarrow\left(\frac{2 n}{\zeta c_{\zeta}}\right)^{n}>\Pi_{i=1}^{n} \sigma_{n-1 ; i}^{\frac{n-\zeta}{n-1}}=\sigma_{n}^{n-\zeta}
\end{aligned}
$$

Therefore, by the assumptions on $f$, we see that $F_{t}(A)>0$.

(7) Noting that $\sum_{i}-\lambda_{i} \frac{\partial F_{t}}{\partial \lambda_{i}}=\sum_{k} \frac{t(n-k) c_{k}}{\left(\begin{array}{l}n \\ k\end{array}\right)} \sigma_{n-k}+n\left(t f+(1-t) c_{0}\right) \sigma_{n}$, it is easy to see using the assumptions on $f$ that the result holds.

(8) Clearly, $\left|\frac{\partial^{2} F_{t, p}}{\partial z^{\mu} \partial \bar{z}^{\mu}}\right| \leq L \sigma_{n}(A) \leq C$.

Now we assume that $F_{t}(A)=1$.

(9) Firstly, we note that $N<\operatorname{tr}(A)=\sum_{i=1}^{n} \lambda_{i} \leq n \lambda_{n}$. As can be seen from [CS17], the above properties imply the result. Indeed, suppose the result is false. Noting that $\theta$ is allowed to depend on $\mu_{i}$, for a given $\mu_{i}$ there is a sequence $\lambda_{i, N}, t_{N}, p_{N}$ (where $N \rightarrow \infty$ ) such that the following inequality holds. (We drop the dependence on $N$ for ease of notation.)

$$
\sum_{i} \frac{\partial F_{t, p}}{\partial \lambda_{i}}(\lambda)\left(\lambda_{i}-\mu_{i}\right)<\frac{1}{N}\left(1-\sum_{i} \frac{\partial F_{t, p}}{\partial \lambda_{i}}(A)\right) .
$$

Since $\frac{\partial F_{t, p}}{\partial \lambda_{i}}(\lambda) \lambda_{i} \geq-C F(A)=-C$,

$$
-C-\frac{1}{N}+\sum_{i}\left(-\frac{\partial F_{t, p}}{\partial \lambda_{i}}(\lambda)\right)\left(\mu_{i}-\frac{1}{N}\right)<0 .
$$

If $-\frac{\partial F_{t, p}}{\partial \lambda_{1}}(\lambda)$ is unbounded (from above) then for sufficiently large $N$, we have a contradiction. Thus $-\frac{\partial F_{t, p}}{\partial \lambda_{1}}(\lambda)>0$ is bounded from above and since 
$-n \lambda_{1} \frac{\partial F_{t, p}}{\partial \lambda_{1}}(\lambda) \geq-\sum_{i} \lambda_{i} \frac{\partial F_{t, p}}{\partial \lambda_{i}}(\lambda) \geq \frac{1}{C}$, we see that $\lambda_{1}>\frac{1}{C^{\prime}}$ for some $C^{\prime}$ independent of $t, p, A$. Therefore, the function $g_{t, p}$ is smoothly controlled (independent of $N)$.

As in [CS17], denote by $\tilde{F}_{t, p}(\lambda)$ the smooth convex function $\lim _{\lambda_{n} \rightarrow \infty} F_{t, p}(\lambda)=$ $g_{t, p}\left(\lambda_{1}, \ldots, \lambda_{n-1}, 0\right)$. By convexity,

$$
\tilde{F}_{t, p}(\mu) \geq \tilde{F}_{t, p}(\lambda)+\sum_{i=1}^{n-1} \frac{\partial \tilde{F}_{t, p}}{\partial \lambda_{i}}(\lambda)\left(\mu_{i}-\lambda_{i}\right) .
$$

Therefore,

$$
\begin{aligned}
\sum_{i} \frac{\partial F_{t, p}}{\partial \lambda_{i}}(\lambda)\left(\lambda_{i}-\mu_{i}\right) \geq & \sum_{i=1}^{n}\left(\frac{\partial F_{t, p}}{\partial \lambda_{i}}(\lambda)-\frac{\partial \tilde{F}_{t, p}}{\partial \lambda_{i}}(\lambda)\right)\left(\lambda_{i}-\mu_{i}\right) \\
& +\tilde{F}_{t, p}(\lambda)-\tilde{F}_{t, p}(\mu) .
\end{aligned}
$$

Since $F_{t, p}(\lambda)=1$ for sufficiently large $N, \tilde{F}_{t, p}\left(\lambda_{i}\right)>1-\tau$ for a small $\tau$. Since $\mu$ satisfies the cone condition, $\tilde{F}_{t, p}(\mu) \leq 1-\delta$ for some $\delta>0$. It is easy to see that

$$
\begin{aligned}
\frac{\partial F_{t, p}}{\partial \lambda_{i}}(\lambda)-\frac{\partial \tilde{F}_{t, p}}{\partial \lambda_{i}}(\lambda) & =-x_{i}^{2}\left(\frac{\partial g_{t, p}\left(x_{1}, \ldots, x_{n}\right)}{\partial x_{i}}-\frac{\partial g_{t, p}\left(x_{1}, \ldots, 0\right)}{\partial x_{i}}\right) \\
& =-x_{i}^{2} x_{n} h_{t, p}(x)
\end{aligned}
$$

where $x_{i}=\frac{1}{\lambda_{i}}$, and $h_{t, p}$ is smooth and uniformly controlled independent of $N$. Therefore, for sufficiently large $N$ (and small $\tau$ ),

$$
\sum_{i} \frac{\partial F_{t, p}}{\partial \lambda_{i}}(\lambda)\left(\lambda_{i}-\mu_{i}\right) \geq \frac{\delta}{2}
$$

Since $\frac{\partial F}{\partial \lambda_{i}}$ is bounded, we arrive at a contradiction between Inequalities 2.23 and 2.18 for large $N$.

(10) Note that $\left|\frac{\partial^{2} F_{t, p}(A)}{\partial \lambda_{i} \partial z^{\mu}}\right| \leq \frac{L \sigma_{n}}{\lambda_{i}}$. Akin to the above properties, one can prove that $-\lambda_{i}^{2} \frac{\partial F}{\partial \lambda_{i}} \geq \frac{\sigma_{n-1 ; i}}{C^{\prime}}$ for some $C^{\prime}$. Now,

$$
\begin{aligned}
\sum_{i} \frac{L \sigma_{n}\left|B_{i \mu}\right|}{\lambda_{i}} & \leq-\sum_{i, \mu} L C^{\prime} \frac{\partial F_{t, p}}{\partial \lambda_{i}}\left|B_{i \mu}\right| \\
& \leq-\frac{C^{\prime \prime}}{\epsilon} \sum_{i} \frac{\partial F_{t, p}}{\partial \lambda_{i}} \lambda_{i}-\epsilon \sum_{i, \mu} \frac{\partial F_{t, p}}{\partial \lambda_{i}} \frac{\left|B_{i \mu}\right|^{2}}{\lambda_{i}} \\
& \leq \frac{C}{\epsilon}-\epsilon \sum_{i, \mu} \frac{\partial F_{t, p}}{\partial \lambda_{i}} \frac{\left|B_{i \mu}\right|^{2}}{\lambda_{i}}
\end{aligned}
$$


The aforementioned lemma is enough to guarantee that Proposition 2.1 holds when $2 \zeta \geq n$ by the method of [PIN19], when followed word-to-word. In fact, such an estimate works for any function $F_{t, p}$ that satisfies the above conditions (along with property 2.10 for $\alpha=0$ ). However, when $\alpha=\frac{1}{2}$, we need to do more work. To this end, we prove a general proposition about estimates.

Proposition 2.2. For any family of PDE of the form $F_{t, p}(A)=1$, where the endomorphism $A$ is given by $A_{k}^{i}:=\chi^{i \bar{j}}\left(\Omega_{\phi_{t}}\right)_{k \bar{j}}$, the solution $\phi_{t}$ (normalised to satisfy $\left.\inf \phi_{t}=0\right)$ satisfies the a priori estimate

$$
\Delta_{\chi} \phi_{t} \leq C\left(1+\left|\nabla_{\chi} \phi_{t}\right|^{2}\right)
$$

if $\left\|\phi_{t}\right\|_{C^{0}}$ is assumed to be uniformly bounded, all the properties in Lemma 2.2 are satisfied by a symmetric function $F_{t, p}$ whose domain $S_{t, p}$ is an open convex cone lying in the set of Hermitian positive-definite matrices, and there is a Kähler metric $\Omega$ in the class $\left[\Omega_{0}\right]$ such that $\chi(p)^{i \bar{j}} \Omega(p)_{k \bar{j}} \in \cap_{0 \leq t \leq 1} S_{t, p}$ for all $p \in M$.

Proof. The following calculations are inspired from similar ones in [CJY20, SZE18].

For a function (akin to $[\mathrm{PS} 09]) \gamma:[0, \infty) \rightarrow \mathbb{R}$ defined as

$$
\gamma(x)=A x-\frac{1}{1+x}+1,
$$

where $A>1$ is a constant to be chosen later, the following properties hold. We suppress the $t$ subscript for $\phi_{t}$ in this proof.

$$
\begin{aligned}
A x & \leq \gamma(x) \leq A x+1 \\
A & \leq \gamma^{\prime}(x) \leq 2 A \\
\gamma^{\prime \prime}(x) & =-\frac{2}{(1+x)^{3}} \leq 0 .
\end{aligned}
$$

Let $G\left(\Omega_{\phi}\right)=-\gamma(\phi)+\ln \left(\lambda_{n}\right)$ where $\lambda_{1} \leq \lambda_{2} \leq \ldots \lambda_{n}$ are the eigenvalues of $A$. Note that $G$ is a continuous function. Suppose the maximum of $G$ is attained at a point $p$. Choose holomorphic normal coordinates for $\chi$ at $p$ such that $\Omega_{\phi}$ is diagonal. In general, $\lambda_{n}$ is not smooth near $p$. The standard remedy is to consider a small constant diagonal matrix $B$ such that $0=B_{n n}<B_{n-1 n-1}<\cdots<B_{11}$ and a new matrixvalued function $\tilde{A}=A-B$. $B$ is chosen to be small enough so that $\tilde{A}$ is still positivedefinite and lies in $S_{t}$ for all points $q$ in a neighbourhood $U$ of $p$. The eigenvalues of $\tilde{A}$ are distinct and are hence smooth in $U$. The function $\tilde{G}=G(\tilde{A})$ continues to achieve a local maximum at $p$. In what follows, the notation $H_{, i}, H_{, i \bar{j}}$ denotes partial derivatives (as opposed to covariant derivatives). At $p$, we differentiate with respect to $z^{i}, \bar{z}^{i}$ to obtain the following.

$$
\begin{aligned}
0 & =\tilde{G}_{, i}(\tilde{A})=-\gamma^{\prime} \phi_{, i}+\frac{1}{\lambda_{n}(\tilde{A})} \frac{\partial \lambda_{n}}{\partial \tilde{A}_{\mu \nu}} \tilde{A}_{\mu \nu, i} \\
& =-\gamma^{\prime} \phi_{, i}+\frac{\left(\Omega_{\phi}\right)_{n \bar{n}, i}}{\lambda_{n}(A)}
\end{aligned}
$$


We drop the $t, p$ subscripts from now onwards. We differentiate again to obtain the following.

$$
\begin{aligned}
0 \geq & -\sum_{i} \frac{\partial F}{\partial \lambda_{i}}(A) \tilde{G}_{, i \bar{i}}(\tilde{A})=\sum_{i}\left(-\frac{\partial F}{\partial \lambda_{i}}(A)\right)\left(-\gamma^{\prime \prime}\left|\phi_{, i}\right|^{2}-\gamma^{\prime} \phi_{, i \bar{i}}-\frac{\left|\left(\Omega_{\phi}\right)_{n \bar{n}, i}\right|^{2}}{\lambda_{n}^{2}(A)}\right. \\
& \left.+\frac{1}{\lambda_{n}(A)} \frac{\partial^{2} \lambda_{n}(\tilde{A})}{\partial \tilde{A}_{p q} \partial \tilde{A}_{r s}}\left(\Omega_{\phi}\right)_{p q, i}\left(\Omega_{\phi}\right)_{r s, \bar{i}}+\frac{\left(\Omega_{\phi}\right)_{n \bar{n}, i \bar{i}}+\lambda_{n}(A) R_{n \bar{n} i \bar{i}}}{\lambda_{n}(A)}\right) .
\end{aligned}
$$

From now onwards, we denote $\lambda_{i}=\lambda_{i}(A)$ and $\tilde{\lambda}_{i}=\lambda_{i}(\tilde{A})=\lambda_{i}-B_{i i}$. Assume that $\lambda_{n}>N$ in order to use property 9 of Lemma 2.2. Assume that $\left|R_{i \bar{i} j \bar{j}}\right| \leq C_{1} \forall i, j$. Using the formulae from [SZE18] we see that

$$
\begin{aligned}
0 \geq & \sum_{i}\left(-\frac{\partial F}{\partial \lambda_{i}}\right)\left(-\gamma^{\prime \prime}\left|\phi_{, i}\right|^{2}-\frac{\left|\left(\Omega_{\phi}\right)_{n \bar{n}, i}\right|^{2}}{\lambda_{n}^{2}}+\frac{\left(\Omega_{\phi}\right)_{n \bar{n}, i \bar{i}}}{\lambda_{n}}\right. \\
& \left.+\frac{1}{\lambda_{n}} \sum_{p<n} \frac{\left|\left(\Omega_{\phi}\right)_{p \bar{n}, i}\right|^{2}+\left|\left(\Omega_{\phi}\right)_{n \bar{p}, i}\right|^{2}}{\lambda_{n}-\tilde{\lambda}_{p}}\right)+\frac{\gamma^{\prime} \theta}{2}\left(1-\sum_{i} \frac{\partial F}{\partial \lambda_{i}}\right)
\end{aligned}
$$

where we assumed that $\frac{\gamma^{\prime} \theta}{2} \geq \frac{A \theta}{2} \geq C_{1}$. Now we compute the derivatives of the PDE $F(A, p)=1$.

$$
\begin{aligned}
F_{, n}+\frac{\partial F}{\partial A_{p q}}\left(A_{p q}\right)_{, n} & =0 \\
\Rightarrow F_{, n}+\sum_{i} \frac{\partial F}{\partial \lambda_{i}}\left(\Omega_{\phi}\right)_{i \bar{i}, n} & =0 .
\end{aligned}
$$

We differentiate again to obtain the following equality.

$$
\begin{aligned}
0= & F_{, n \bar{n}}+\sum_{i} 2 \operatorname{Re}\left(\frac{\partial F_{, n}}{\partial \lambda_{i}}\left(\Omega_{\phi}\right)_{i \bar{i}, \bar{n}}\right)+\sum_{p, q, r, s} \frac{\partial^{2} F}{\partial A_{p q} \partial A_{r s}}\left(\Omega_{\phi}\right)_{p \bar{q}, n}\left(\Omega_{\phi}\right)_{r \bar{s}, n} \\
& +\sum_{i} \frac{\partial F}{\partial \lambda_{i}}\left(\left(\Omega_{\phi}\right)_{i \bar{i}, n \bar{n}}+R_{\bar{i} \bar{i} \bar{n}} \lambda_{i}\right)
\end{aligned}
$$

We substitute the fourth order term from Equation 2.31 into 2.29, and use the assumptions of Lemma 2.2 to get the following.

$$
\begin{aligned}
0 \geq & \frac{\gamma^{\prime} \theta}{2}\left(1-\sum_{i} \frac{\partial F}{\partial \lambda_{i}}\right)+\sum_{i} \frac{\partial F}{\partial \lambda_{i}}\left(\gamma^{\prime \prime}\left|\phi_{, i}\right|^{2}+\frac{\left|\left(\Omega_{\phi}\right)_{n \bar{n}, i}\right|^{2}}{\lambda_{n}^{2}}\right)+\frac{F_{, n \bar{n}}}{\lambda_{n}} \\
& +\frac{1}{\lambda_{n}}\left(C_{1} \sum_{i} \lambda_{i} \frac{\partial F}{\partial \lambda_{i}}-C+\frac{1}{2} \sum_{i} \frac{\partial F}{\partial \lambda_{i}} \frac{\left|\left(\Omega_{\phi}\right)_{i \bar{i}, n}\right|^{2}}{\lambda_{i}}-\sum_{i, j} \frac{\partial F}{\partial \lambda_{i}} \frac{\left|\left(\Omega_{\phi}\right)_{i \bar{j}, n}\right|^{2}}{\lambda_{j}}\right. \\
& \left.+\frac{1}{2} \sum_{i} \frac{\partial F}{\partial \lambda_{i}} \frac{\left|\left(\Omega_{\phi}\right)_{i \bar{i}, n}\right|^{2}}{\lambda_{i}}\right)
\end{aligned}
$$




$$
\geq \frac{\gamma^{\prime} \theta}{2}\left(1-\sum_{i} \frac{\partial F}{\partial \lambda_{i}}\right)-\frac{C}{\lambda_{n}}+\frac{\partial F}{\partial \lambda_{n}} \frac{\left|\left(\Omega_{\phi}\right)_{n \bar{n}, n}\right|^{2}}{\lambda_{n}^{2}} .
$$

At this juncture, we use Equation 2.27 in 2.32 to get the following inequality.

$$
0 \geq \frac{\gamma^{\prime} \theta}{2}-\frac{C}{\lambda_{n}}+\lambda_{n} \frac{\partial F}{\partial \lambda_{n}} \frac{\left(\gamma^{\prime}\right)^{2}\left|\phi_{, n}\right|^{2}}{\lambda_{n}} .
$$

Assume that $\lambda_{n}>1$ and that $A \frac{\theta}{4} \geq C$. Note that $-C \leq \lambda_{n} \frac{\partial F}{\partial \lambda_{n}} \leq 0$ since

$$
-C \leq \sum_{i} \lambda_{i} \frac{\partial F}{\partial \lambda_{i}}
$$

by assumption. Thus,

$$
0 \geq \frac{A \theta}{4}-\frac{4 C A^{2}\left|\phi_{, n}\right|^{2}}{\lambda_{n}} .
$$

Therefore, $\lambda_{n} \leq C\left(1+|\nabla \phi|^{2}\right)$.

Using Proposition 2.2 and Lemma 2.2, we see that in our case, indeed

$$
\Delta \phi \leq C\left(1+|\nabla \phi|^{2}\right)
$$

Now, Proposition 5.1 of [CJY20] (which is based on a blow-up argument) shows that $\Delta \phi \leq C$, as desired.

At this point, we note that since $F_{t}(A)$ is convex on a convex open set, the complex version of Evans-Krylov theory akin to [SIU86] would have implied the $C^{2, \alpha}$ a priori estimates on $\phi_{t}$ if $f$ was constant. In our case, despite $f$ not being a constant, we can still get $C^{2, \alpha}$ estimates on $\phi_{t}$ using a blow-up argument (like in the proof of Lemma 6.2 in [CJY20] for instance). Using Schauder theory, we can get $C^{k, \alpha}$ bounds (depending on $k$ ) for every $k$. Applying the Arzela-Ascoli theorem and choosing a diagonal subsequence, we get closedness of the set of $t \in[0,1]$ such that Equation 2.8 has a smooth solution. Therefore, we have a smooth solution at $t=1$.

\section{Concentration of Mass}

We begin with a definition of what it means for a possibly singular $(1,1)$ current to satisfy the cone condition. Let $\rho:[0,1] \rightarrow \mathbb{R}$ be a smooth function such that

$$
\left|\mathbb{S}^{2 n-1}\right| \int_{0}^{1} \rho(t) t^{2 n-1} d t=1
$$


where $\left|\mathbb{S}^{2 n-1}\right|$ is the Lebesgue measure of the unit sphere in $\mathbb{C}^{n}$. For any $1>\delta>0$, and any $L^{1}$-function $\varphi: B_{1}(0) \rightarrow \mathbb{R}$ on the unit ball $B_{1}(0)$, the $\delta$-mollification is defined to be

$$
\varphi_{\delta}(x)=\delta^{-2 n} \int_{B_{1}(0)} \varphi(x-y) \rho\left(\frac{|y|}{\delta}\right) d y .
$$

Then we have the following definition from [CHEN21].

Definition 1. Let $\Theta$ be a closed, positive $(1,1)$ current. We say that $\Theta$ satisfies the $(\varepsilon-)$ uniform cone condition

$$
n(1-\varepsilon) \Theta^{n-1}-\sum_{k=1}^{n-1} c_{k} k \chi^{n-k} \Theta^{k-1} \geq 0,
$$

if for any coordinate chart $U$ with $\left.\Theta\right|_{U}=\sqrt{-1} \partial \bar{\partial} \varphi_{U}$, on $U_{\delta}:=\{x \in M \mid B(x, \delta) \subset$ $U\}$ (where $B(x, \delta)$ is a coordinate Euclidean ball of radius $\delta$ centred at $x$ ), we have

$$
n(1-\varepsilon)\left(\sqrt{-1} \partial \bar{\partial} \varphi_{U, \delta}\right)^{n-1}(x)-\sum_{k=1}^{n-1} c_{k} k \chi_{0}^{n-k}\left(\sqrt{-1} \partial \bar{\partial} \varphi_{U, \delta}\right)^{k-1}(x) \geq 0,
$$

for any Kähler metric $\chi_{0}$ on $U$ with constant coefficients satisfying $\chi_{0} \leq \chi$ on $B(x, \delta)$. Here $\varphi_{U, \delta}$ are the mollifications of $\varphi_{U}$ as above.

If there exist strictly positive sequences $\varepsilon_{i}, \mu_{i} \rightarrow 0$ such that $\Theta+\mu_{i} \chi$ satisfies the $\left(\varepsilon_{i^{-}}\right)$uniform cone condition, then we see that $\Theta$ satisfies the degenerate cone condition.

REMARK 3.1. If $\Theta$ is a smooth form, clearly the definitions above coincide with the usual pointwise definition. In particular, the degenerate cone condition boils down to the pointwise condition $n \Theta^{n-1}-\sum_{k=1}^{n-1} c_{k} k \chi^{n-k} \Theta^{k-1} \geq 0$.

The main goal of this section is to prove the following "mass concentration" result.

Theorem 3.1. Let $(M, \chi)$ be a connected Kähler manifold and $\Omega_{0}$ another Kähler metric. Suppose for all $t>0$, there exists a $\hat{\Omega}_{t} \in(1+t)\left[\Omega_{0}\right]$ such that

$$
n \hat{\Omega}_{t}^{n-1}-\sum_{k=1}^{n-1} c_{k} k \chi^{n-k} \hat{\Omega}_{t}^{k-1}>0 .
$$

Then for any divisor $Y$, there exists a $\beta_{Y}>0$ and a current $\Theta \in\left[\Omega_{0}\right]$ such that $\Theta \geq \beta_{Y}[Y]$ and $\Theta$ satisfies the degenerate cone condition in the sense above. If $G$ is a connected compact Lie group acting on $M$ such that $\chi$ and $Y$ are $G$-invariant, then $\Theta$ can be chosen to be $G$-invariant as well. 
Proof. The proof is essentially a combination of the ideas in [CHEN21] and [DP01]. Suppose that $M$ be covered by coordinate balls $\left\{B_{j}\right\}_{j=1}^{N}$, and that $Y$ is given by the vanishing of coordinate functions $\left\{g_{j}\right\}$ on $B_{j}$. Let $\left\{\theta_{j}\right\}$ be the partition of unity subordinate to $B_{j}$. For $0 \leq t<<1$, define

$$
\psi_{t}=\log \left(\sum_{j}\left(\theta_{j} \sum_{k}\left|g_{j, k}\right|^{2}\right)+t^{2}\right), \chi_{t}=\chi+A^{-1} \sqrt{-1} \partial \bar{\partial} \psi_{t},
$$

where we pick a suitable $A>>1$ below. For $t$ small enough, $\chi_{t}$ is a smooth Kähler form on $M$ which concentrates near $Y$, and $\chi_{t}>\left(1-C A^{-1}\right) \chi$ for some fixed $C$ (independent of $t$ ). Now consider the following equation:

$$
\Omega_{t}^{n}=\sum_{k=1}^{n-1} c_{k} \chi^{n-k} \Omega_{t}^{k}+f_{t} \chi^{n}
$$

where $\Omega_{t} \in(1+t)\left[\Omega_{0}\right]$ and

$$
f_{t}=\frac{\chi_{t}^{n}}{\chi^{n}}-1+A_{t}
$$

and the constant $A_{t}$ is chosen so that

$$
\int_{M} \Omega_{t}^{n}=\sum_{k=1}^{n-1} \int_{M} c_{k} \chi^{n-k} \Omega_{t}^{k}+\int_{M} f_{t} \chi^{n}
$$

Note that $A_{t}>0$ for all $t$. Also, for $\varepsilon<<1$, we have that $f_{t}>f_{m}$, and hence by Theorem 2.1 , there is a solution $\Omega_{t} \in(1+t)\left[\Omega_{0}\right]$ to $(3.2)$ satisfying the cone condition

$$
n \Omega_{t}^{n-1}-\sum_{k=1}^{n-1} c_{k} k \chi^{n-k} \Omega_{t}^{k-1}>0
$$

Now the family of currents $\Omega_{t}$ is bounded in mass since

$$
\int_{M} \Omega_{t} \wedge \chi^{n-1}=(1+t)\left[\Omega_{0}\right] \cdot\left[\chi^{n-1}\right]<C .
$$

Define $\tilde{\Omega}_{t}$ as the average of $\Omega_{t}$ over $G$. Since the cohomology class of $\Omega_{t}$ does not change by averaging over a compact connected Lie group, the family $\tilde{\Omega}_{t}$ is also bounded in mass. Hence, there exists a sequence $t_{i} \rightarrow 0^{+}$such that $\tilde{\Omega}_{t_{i}, \varepsilon} \rightarrow \Theta$, where $\Theta$ is a non-negative current in the class $\alpha$. Clearly, $\Theta$ is $G$-invariant. We now show that $\Theta \geq \beta_{Y}[Y]$ for some $\beta_{Y}>0$ following the line of argument in [DP01]. First we have the following simple observation. 
Lemma 3.1. For any neighbourhood $U$ of a point $y \in Y$, there exist constants $\delta_{U}, t_{U}>0$ such that for all $t<t_{U}$ the following inequality holds.

$$
\int_{U \cap V_{t}} \Omega_{t} \wedge \chi^{n-1}>\delta_{U}
$$

where $V_{t}=\left\{\psi_{0}<\log t\right\}$.

Proof. Let $0 \leq \lambda_{1}(z) \leq \ldots \leq \lambda_{n}(z)$ be the eigenvalues of $\Omega_{t}$ with respect to $\chi_{t}$. Since $\Omega_{t}^{n-1} \wedge \chi_{t} \geq \lambda_{2} \ldots \lambda_{n} \chi_{t}^{n}$, we have that

$$
\int_{M} \lambda_{2} \cdots \lambda_{n} \chi_{t}^{n} \leq \int_{M} \Omega_{t}^{n-1} \wedge \chi_{t}=(1+t)^{n-1}\left(\Omega_{0}^{n-1}\right) \cdot[\chi] \leq C .
$$

In particular, for any $\delta>0$, if $E_{\delta}:=\left\{z \in M \mid \lambda_{2}(x) \cdots \lambda_{n}(x)>C \delta^{-1}\right\}$, then

$$
\int_{E_{\delta}} \chi_{t}^{n} \leq \delta
$$

By Lemma 2.1 in [DP01], since $\chi<2 \chi_{t}$, we have that

$$
\int_{E_{\delta}} \chi_{t} \wedge \chi^{n-1}<2^{n-1} \int_{E_{\delta}} \chi_{t}^{n}<2^{n-1} \delta
$$

Next, by [DP01, Lemma 2.1(iii)], there exists a $\delta(U)$ such that for all $t<<1$,

$$
\begin{aligned}
\int_{U \cap V_{t} \cap E_{\delta}^{c}} \chi_{t} \wedge \chi^{n-1} & =\int_{U_{\cap} V_{t}} \chi_{t} \wedge \chi^{n-1}-\int_{U \cap V_{t} \cap E_{\delta}} \chi_{t} \wedge \chi^{n-1} \\
& \geq \delta(U)-2^{n-1} \delta=\frac{3}{4} \delta(U),
\end{aligned}
$$

where we let $\delta:=\frac{\delta(U)}{2^{n+1}}$. On the other hand, from our equation, $\Omega_{t}^{n} \geq f_{t} \chi^{n} \geq \chi_{t}^{n}-\chi^{n}$. So if $z \in E_{\delta}^{c}$, then

$$
\Omega_{t}(z) \geq \frac{\delta}{C}\left(1-\frac{\chi^{n}(z)}{\chi_{t}^{n}(z)}\right) \chi_{t}(z)
$$

Integrating,

$$
\begin{aligned}
\int_{U \cap V_{t}} \Omega_{t} \wedge \chi^{n-1} & \geq \int_{U \cap V_{t} \cap E_{\delta}^{c}} \Omega_{t} \wedge \chi^{n-1} \\
& \geq \frac{\delta}{C}\left(\int_{U \cap V_{t} \cap E_{\delta}^{c}} \chi_{t} \wedge \chi^{n-1}-\int_{U \cap V_{t} \cap E_{\delta}^{c}} \frac{\chi^{n}}{\chi_{t}^{n}} \chi_{t} \wedge \chi^{n-1}\right) \\
& \geq \frac{\delta}{C}\left(\frac{3 \delta(U)}{4}-\int_{U \cap V_{t} \cap E_{\delta}^{c}} \frac{\chi^{n}}{\chi_{t}^{n}} \chi_{t} \wedge \chi^{n-1}\right) .
\end{aligned}
$$

For the second term we estimate

$$
\int_{U \cap V_{t} \cap E_{\delta}^{c}} \frac{\chi^{n}}{\chi_{t}^{n}} \chi_{t} \wedge \chi^{n-1}<2^{n-1} \int_{U \cap V_{t}} \chi^{n}
$$


and hence it can be made smaller than $\delta(U) / 4$ if we choose $t<t_{U}$ for some $t_{U}$ depending only on $\chi$ and $Y$. The proof of the Lemma is then completed by choosing $\delta_{U}=\frac{\delta \delta(U)}{2 C}$.

Now, as in [DP01], consider the current $\mathbf{1}_{Y} \Theta$. By the Skoda-El Mir extension theorem, $\mathbf{1}_{Y} \Theta$ is a closed, non-negative $(1,1)$ current supported on $Y$. By standard support theorems, $\mathbf{1}_{Y} \Theta=\sum_{i} \beta_{i}\left[Y_{i}\right]$ for some $\beta_{i} \geq 0$, where $Y_{i}$ are the irreducible components of $Y$. It can be easily seen using Lemma 3.1 that $\beta_{i}>0 \forall i$. Hence $\Theta \geq \beta_{Y}[Y]$ on all of $M$, where $\beta_{Y}$ can be taken as $\min _{i} \beta_{i}$.

Finally, we verify the degenerate cone condition. To this end, we need the following lemma.

Lemma 3.2. Let $\chi(x), \Omega(x)$ be smooth Kähler forms at a point $x \in M$. Let $\alpha$ be a fixed smooth Kähler metric on $M$ such that $\chi(x) \leq C_{\chi} \alpha(x)$ for some $C_{\chi}>0$. Assume that the following degenerate cone condition is met at $x$.

$$
n \Omega^{n-1}(x)-\sum_{k=1}^{n-1} c_{k} k \chi^{n-k}(x) \Omega^{k-1}(x) \geq 0 .
$$

Let $\beta>0$. There exists an $\epsilon>0$ depending only only on the coefficients $c_{k}$ and the constants $C_{\chi}, \beta$ such that $\Omega^{\prime}(x)=\Omega(x)+2 \beta \alpha(x)$ satisfies the $\epsilon$-uniform cone condition.

$$
n(1-\epsilon)\left(\Omega^{\prime}\right)^{n-1}(x)-\sum_{k=1}^{n-1} c_{k} k \chi^{n-k}(x)\left(\Omega^{\prime}\right)^{k-1}(x) \geq 0 .
$$

Proof. Choose coordinates near $x$ such that $\chi(x)$ is Euclidean and $\Omega(x)$ is diagonal with eigenvalues $\lambda_{i}$. Then the cone condition can be written as

$$
1 \geq \sum_{k=1}^{n-1} \frac{c_{k}}{\left(\begin{array}{l}
n \\
k
\end{array}\right)} S_{n-k ; j}\left(\frac{1}{\lambda}\right) \forall 1 \leq j \leq n .
$$

Note that $\Omega^{\prime}(x) \geq \Omega(x)+2 \beta \frac{\chi(x)}{C_{\chi}}$. Let $\tilde{\lambda}_{i}=\lambda_{i}+\frac{\beta}{C_{\chi}} \geq \gamma$ where $\gamma=\frac{\beta}{C_{\chi}}$. By monotonicity, $\left\{\tilde{\lambda}_{i}\right\}$ also satisfies Inequality 3.5 . Let $\lambda_{i}^{\prime}=\tilde{\lambda}_{i}+\gamma$. If we prove that $\left\{\lambda_{i}^{\prime}\right\}$ satisfies the $\epsilon$-uniform cone condition for some $\epsilon$, then again by monotonicity, so does $\Omega^{\prime}$.

To this end, we first note that

$$
\frac{1}{\lambda_{i}^{\prime}} \geq \frac{1}{2 \tilde{\lambda}_{i}} \forall 1 \leq i \leq n
$$

Define

$$
B:=\sum_{k=1}^{n-1} \frac{c_{k}}{\left(\begin{array}{l}
n \\
k
\end{array}\right)} S_{n-k ; j}\left(\frac{1}{\tilde{\lambda}}\right) .
$$


Using 3.6, $\forall 1 \leq j \leq n$ we see that the following holds.

$$
\begin{aligned}
\sum_{k=1}^{n-1} \frac{c_{k}}{\left(\begin{array}{l}
n \\
k
\end{array}\right)} S_{n-k ; j}\left(\frac{1}{\lambda^{\prime}}\right) \leq & \sum_{k=1}^{n-1} \frac{c_{k}}{\left(\begin{array}{l}
n \\
k
\end{array}\right)} S_{n-k ; j}\left(\frac{1}{\tilde{\lambda}}\right) \\
& +\sum_{k=1}^{n-1} \frac{c_{k}}{\left(\begin{array}{l}
n \\
k
\end{array}\right)}\left(S_{n-k ; j}\left(\frac{1}{\lambda^{\prime}}\right)-S_{n-k ; j}\left(\frac{1}{\tilde{\lambda}}\right)\right) \\
\leq & B-\sum_{k=1}^{n-1} \frac{c_{k} \gamma^{n-k}}{2^{n-k}\left(\begin{array}{l}
n \\
k
\end{array}\right)} S_{n-k ; j}\left(\frac{1}{\tilde{\lambda}^{2}}\right) \\
\leq & B-\frac{B^{2}}{C}
\end{aligned}
$$

where $C>4$ is some positive constant depending only on the coefficients $c_{k}$, and the constants $n, \gamma$. Since $0<B<1$, we see that for $\epsilon=\frac{1}{C}$, the above expression is $\leq 1-\epsilon$, as desired.

Let $U$ be a coordinate neighbourhood, and $\left.\Theta\right|_{U}=\sqrt{-1} \partial \bar{\partial} \varphi,\left.\tilde{\Omega}_{t}\right|_{U}=\sqrt{-1} \partial \bar{\partial} \varphi_{t}$. Let $\varphi_{\delta}$ and $\varphi_{t, \delta}$ be the convolutions of $\varphi$ and $\varphi_{t}$ with the standard molifier. Firstly, recall that the $(\varepsilon)$-cone condition for a smooth positive form $\gamma$ can be written as

$$
\sum_{k=1}^{n-1} c_{k} \frac{1}{\left(\begin{array}{l}
n \\
k
\end{array}\right)} S_{n-k ; i}\left(\frac{1}{\lambda_{\gamma}}\right) \leq 1-\varepsilon,
$$

where $\lambda_{\gamma}$ are the eigenvalues of $\gamma$ with respect to $\chi$. Let $\chi_{0}$ be a constant coefficient form in $U$ such that $\chi \geq \chi_{0}$ on $B_{\delta}(x)$. Choose a strictly positive sequence $\mu_{i} \rightarrow 0$. By Lemma 3.2 we see that there exists a corresponding sequence of $\varepsilon_{i} \rightarrow 0$ such that $\sqrt{-1} \partial \bar{\partial} \varphi_{t}+\mu_{i} \chi$ satisfies the $\left(\varepsilon_{i}-\right)$ uniform cone condition. By monotonicity, $\sqrt{-1} \partial \bar{\partial} \varphi_{t}+\mu_{i} \chi$ satisfy the $\left(\varepsilon_{i}-\right)$ uniform cone condition at $x$ with $\chi$ replaced with $\chi_{0}$. Since averaging over a group and convolutions are basically convex linear combinations and the expression on the left-hand side of 3.8 is convex, using Jensen's inequality we see that $\sqrt{-1} \partial \bar{\partial} \varphi_{t, \delta}+\mu_{i} \chi, \delta$ satisfies the degenerate cone condition at $x$ with $\chi_{0}$ replacing $\chi$. Moreover, since $\Omega_{t} \rightarrow \Theta$ weakly, and convolution is basically testing against a smooth function, we see that $\sqrt{-1} \partial \bar{\partial} \varphi_{t, \delta}+\mu_{i} \chi, \delta$ converges pointwise to $\sqrt{-1} \partial \bar{\partial} \varphi_{\delta}+\mu_{i} \chi \chi, \delta$ as $t \rightarrow 0$. Hence $\sqrt{-1} \partial \bar{\partial} \varphi_{\delta}+\mu_{i} \chi, \delta$ satisfies the $\left(\varepsilon_{i}-\right)$ uniform cone condition at $x$. Therefore, $\Theta$ satisfies the degenerate cone condition as in Definition 1.

\section{Proof of Theorem 1.3}

In this section, we denote by $E_{c}(T)$, the Lelong subvariety $\{x \mid \nu(T, x) \geq c>0\}$. Note that if $T$ is $G$-invariant, then so is $E_{c}(T)$ for all $c$. Our goal is is to prove Theorem 1.3. First we need the following gluing proposition. 
Proposition 4.1. Let $(M, \chi)$ be a compact Kähler manifold, $\left[\Omega_{0}\right]$ be another Kähler class, $Y$ be an ample divisor. Let $0<\beta<1$, and $0<\varepsilon_{4.1}<\frac{\beta}{1000 c_{n}}$, where $c_{n}$ is the dimensional constant defined below. Suppose $T \geq \beta[Y]$ is a positive current in $\left[\left(1-\varepsilon_{4.1}\right) \Omega_{0}\right]$ satisfying the strict cone condition on $M \backslash Y$. Then there exists a $c_{4.1}$ with the following property: If $U$ is a neighbourhood of $Z:=E_{c_{4} .1}(T) \cup Y$ with a Kähler form $\theta=\left.\Omega_{0}\right|_{U}+\sqrt{-1} \partial \bar{\partial} \psi_{U}$ satisfying the cone condition on $U$, then there exists a Kähler form $\hat{\Omega}_{0}=\Omega_{0}+\sqrt{-1} \partial \bar{\partial} \hat{\psi}$ satisfying the cone condition, i.e.,

$$
n \hat{\Omega}_{0}^{n}-\sum_{k=1}^{n-1} c_{k} k \chi^{n-k} \hat{\Omega}_{0}^{k-1}>0 .
$$

Proof. We follow the line of argument in [CHEN21] closely, with one necessary addition. The basic idea is to cover the manifolds with balls of a small but definite size on which the current can be written as the $\sqrt{-1} \partial \bar{\partial}$ of some potentials, and then to glue these potentials to $\psi_{U}$ using the modification of the Richberg technique [RICH68] due to Blocki-Kolodziej [BK07], and its improvement (to allow small Lelong numbers) in [CHEN21]. The additional complication in our case, as opposed to the method in [CHEN21], is that $T$ satisfies the cone condition only on $M \backslash Y$, while in [CHEN21], the cone condition is satisfied on all of $M$. So we need to make sure that during the gluing process, the metric $\theta$ is unchanged in a neighbourhood of $Y$.

- We cover $M$ with a finite number of balls $B_{r}^{i}=B_{r}\left(x_{i}\right)$ of radius $r<1$ (with respect to $\Omega_{0}$ ) centred at $x_{i}$ such that the following conditions hold:

(1) For every $z \in Y$, there exists an $i$, such that $B_{r / 4}(z) \subset B_{r}^{i}$, where $B_{r / 4}(z)$ is the $\Omega_{0}$-ball of radius $\frac{r}{4}$ around $z$.

(2) On $B_{2 r}^{i}:=B_{2 r}^{i}\left(x_{i}\right)$, we have $\Omega_{0}=\sqrt{-1} \partial \bar{\partial} \varphi_{0}^{i}$, and

$$
\begin{gathered}
\chi_{0}^{i} \leq \chi \leq\left(1+\frac{\varepsilon_{4.1}}{1000 n^{10 n}}\right) \chi_{0}^{i} \\
\left.\left|\varphi_{0}^{i}-\right| z\right|^{2} \mid<\varepsilon_{4.1} r^{2},
\end{gathered}
$$

where $\chi_{0}^{i}$ is a Kähler form on $B_{2 r}^{i}$ with constant coefficients. Moreover, choose the holomorphic coordinates on the ball to be such that $\Omega_{0}$ is within $1+\frac{1}{1000}$ of the Euclidean metric.

It is clear that the second condition can be achieved by choosing a sufficiently refined cover. For the first condition, we simply include a finitely many balls $\left\{B_{r}^{i}:=B_{r}\left(z_{i}\right)\right\}_{i=1}^{N}$ centred at points $z_{i} \in Y$ such that $Y \subset \cup_{i=1}^{N} B_{r / 4}\left(z_{i}\right)$.

Next we let $\varphi_{\delta}^{i}$ be the smoothening (by convolution) of $\varphi^{i}:=\varphi_{T}+\left(1-\varepsilon_{4.1}\right) \varphi_{0}^{i}$, where $T=\left(1-\varepsilon_{4.1}\right) \Omega_{0}+\sqrt{-1} \partial \bar{\partial} \varphi_{T}$. By our hypothesis, $T=\sqrt{-1} \partial \bar{\partial} \varphi^{i}$ satisfies the (strict) cone condition on $M \backslash Y$. By convexity, it then follows that $\sqrt{-1} \partial \bar{\partial} \varphi_{\delta}^{i}$ also satisfies the cone condition

$$
n\left(\sqrt{-1} \partial \bar{\partial} \varphi_{\delta}^{i}\right)^{n-1}-\sum_{k=1}^{n-1} c_{k} k \chi^{n-k}\left(\sqrt{-1} \partial \bar{\partial} \varphi_{\delta}^{i}\right)^{k-1}>0
$$


at every $x \in B_{r}^{i}$ so long as $B_{\delta}(x)$ does not intersect $Y$.

- Recall that the Lelong number at level $\delta<r / 4$ is defined by

$$
\nu^{i}(x, \delta)=\frac{\hat{\varphi}_{\frac{r}{4}}^{i}(x)-\hat{\varphi}_{\delta}^{i}}{\log \frac{r}{4}-\log \delta},
$$

where $\hat{\varphi}_{\delta}^{i}(x)=\sup _{B_{\delta}^{i}} \varphi^{i}$. One can show that $\nu^{i}(x, \delta)$ is increasing in $\delta$, and the Lelong number of $T$ at $x$ (which of course is independent of the potential) is given by

$$
\nu(T, x)=\lim _{\delta \rightarrow 0^{+}} \nu^{i}(x, \delta) .
$$

We now let $c_{4.1}:=c_{n} \varepsilon_{4.1} r^{2}$, where

$$
c_{n}=\frac{2}{\left(\left|\mathbb{S}^{2 n-1}\right| \int_{0}^{1} \log t^{-1} \rho(t) t^{2 n-1} d t+\frac{3^{2 n-1}}{2^{2 n-3}}\right)} .
$$

We let $Z:=E_{c_{4.1}} \cup Y$. By our choice of $\varepsilon_{4.1}$, we have that $c_{4.1}<\frac{\beta}{1000}$, and so in particular, $Y \subset E_{c_{4.1}}$. Also note that our constant is $10 \epsilon_{4.5}$, where $\epsilon_{4.5}$ is the constant in Proposition 4.1 in [CHEN21]. Now let $\overline{U^{\prime}} \subset U$. For $\delta$ small (in particular smaller than $\delta_{0}$ in Lemma 4.1 below), if we define $\hat{\psi}$ to be the regularised maximum of $\psi_{U}+\frac{{ }^{c} 4.1}{4} \log \delta$ on $\overline{U^{\prime}}$ and $\varphi_{\delta}^{i}-\varphi_{0}^{i}$, then by [CHEN21, Proposition 4.1], $\hat{\psi}$ will be a smooth $\Omega_{0}$-PSH function. We let $\hat{\Omega}=\Omega_{0}+\sqrt{-1} \partial \bar{\partial} \hat{\psi}$. To complete the proof of the proposition we need to make sure that the cone condition is satisfied. Recall that $T$ satisfies the cone condition on $M \backslash Y$, and hence the smoothening $\sqrt{-1} \partial \bar{\partial} \varphi_{\delta}^{i}$ satisfies the cone condition at $x$ if $B_{\delta}(x)$ does not intersect $Y$. On the other hand $\theta$ satisfies the cone condition everywhere in $U$. If $B_{\delta}(x)$ does intersect $Y$ for some $\delta<\delta_{0}$, where $\delta_{0}$ is as in Lemma 4.1 below, then in particular $d_{\Omega_{0}}(x, Y)<4 \delta$, and so by Lemma 4.1, $\nu^{i}(x, \delta)>c_{4.1} / 2$ for some $i$. Once again by [CHEN21, Proposition $4.1]$,

$$
\max _{B_{r}^{i}}\left(\varphi_{\delta}^{i}-\varphi_{0}^{i}\right) \leq \inf _{\bar{U}^{\prime}}\left(\psi_{U}+\frac{c_{4} .1}{4} \log \delta-\varepsilon_{4.1} r^{2}\right)
$$

In particular, $\hat{\psi}(x)=\psi_{U}(x)+\frac{{ }^{c} 4.1}{4} \log \delta$, and hence $\hat{\Omega}_{0}$ satisfies the cone condition at $x$.

Lemma 4.1. There exists a $\delta_{0}<r / 4$, such for all $\delta<\delta_{0}$, there exists $U_{5 \delta} \subset \bar{U}_{5 \delta} \subset U^{\prime}$, an $\Omega_{0}$-tubular neighbourhood of $Y$ of size $5 \delta$, with the property that for all $x \in U_{5 \delta}$, there exists an $i$ such that $x \in B_{r}^{i}$ and $\nu^{i}(x, \delta)>c_{4.1} / 2$.

Proof. For any plurisubharmonic function $\varphi$, we let $\nu_{\varphi}(x):=\nu(\sqrt{-1} \partial \bar{\partial} \varphi, x)$, and let $\nu_{\varphi}(x, \delta)$ be the corresponding Lelong number at level $\delta$. In each of the balls $B_{2 r}\left(x_{i}\right)$ 
(where $x_{i} \in Y$ ), assume that $Y$ is given by $f_{i}=0$ where $f_{i}$ is an analytic function. Let $\phi_{1}^{i}=\frac{\beta}{10} \ln \left|f_{i}\right|^{2}$ and $\phi_{2}^{i}=\varphi^{i}-\phi_{1}^{i}$.

Note that $\nu_{\varphi^{i}}(x) \geq \nu_{\phi_{1}^{i}}(x)$, since $T \geq \beta[Y]$. Assume $\delta<r / 4<1$. Now, since $\left(\widehat{\psi_{1}+\psi_{2}}\right)_{\delta} \leq \widehat{\left(\psi_{1}\right)_{\delta}}+\widehat{\left(\psi_{2}\right)_{\delta}}$ for any two upper-semicontinuous functions $\psi_{1}, \psi_{2}$, we see that the following holds.

$$
\begin{aligned}
\nu_{\varphi^{i}}(x, \delta)= & \frac{\widehat{\varphi_{r / 4}^{i}}(x)-\widehat{\varphi_{\delta}^{i}}(x)}{\ln (r / 4)-\ln (\delta)} \geq \frac{\widehat{\varphi_{r / 4}^{i}}(x)}{\ln (r / 4)-\ln (\delta)}-\frac{\widehat{\left(\phi_{1}^{i}\right)_{r / 4}}(x)}{\ln (r / 4)-\ln (\delta)}-\frac{\widehat{\left(\phi_{2}^{i}\right)_{r / 4}}(x)}{\ln (r / 4)-\ln (\delta)} \\
& +\nu_{\phi_{1}^{i}}(x, \delta)+\nu_{\phi_{2}^{i}}(x, \delta) .
\end{aligned}
$$

Note that $\nu_{\phi_{2}^{i}}(x, \delta) \geq \nu_{\phi_{2}^{i}}(x)>0$ because the $\delta$-Lelong number increases with $\delta$. Moreover as $\delta \rightarrow 0$, the first three terms go to zero uniformly (in $x, i$ ), and so if $\delta_{0}$ is sufficiently small, then for every $i$,

$$
\nu_{\varphi^{i}}(x, \delta) \geq-\frac{\beta}{100000}+\nu_{\phi_{1}^{i}}(x, \delta) .
$$

For an even smaller $\delta_{0}$,

$$
\nu_{\varphi^{i}}(x, \delta) \geq-\frac{\beta}{5000}+\frac{\beta}{11} \frac{\sup _{B_{x, \delta}}\left(\ln \left(\left|f_{i}\right|^{2}\right)\right)}{\ln (\delta)}
$$

Now we use the assumption that $x \in U_{5 \delta_{0}}$. Hence, there exists a point $z_{x} \in Y$ such that $d_{\Omega_{0}}\left(x, z_{x}\right) \leq 5 \delta_{0}$. This point is at a Euclidean distance (in some coordinate chart $\left.B_{2 r}\left(x_{j}\right)\right)$ of at most $6 \delta_{0}$ from $z_{x}$. Note that $f_{j}\left(z_{x}\right)=0$. Hence,

$$
\left|f_{j}(x)-f_{j}\left(z_{x}\right)\right|^{2} \leq C\left|x-z_{x}\right|^{2} \leq C 36 \delta^{2},
$$

where $C$ depends on $\max _{\bar{B}_{r}\left(x_{j}\right)}\left|\nabla_{\Omega_{0}} f_{j}\right|^{2}$. Hence, for a sufficiently small $\delta_{0}$, Inequality 4.4 implies the following estimate that completes the proof.

$$
\nu_{\varphi^{j}}(x, \delta) \geq \frac{\beta}{20}>\frac{c_{4.1}}{2}
$$

In what follows it is convenient to adopt the following notation: For $m<n$, and $j=0,1, \cdots, m-1$, we set

$$
b_{j}:=\frac{c_{j+n-m}\left(\begin{array}{c}
j+n-m \\
n-m
\end{array}\right)}{\left(\begin{array}{c}
n \\
m
\end{array}\right)} .
$$

Lemma 4.2. Let $Z$ be a smooth $m$-dimensional sub-variety of $M$. Suppose there exists a Kähler metric $\omega_{Z}=\left.\Omega_{0}\right|_{Z}+\sqrt{-1} \partial \bar{\partial} \psi_{Z}$ on $Z$ such that

$$
\left(1-\frac{\varepsilon}{2}\right) \omega_{Z}^{m}-\sum_{j=0}^{m-1} b_{j} \chi^{m-j} \omega_{Z}^{j}>0,
$$


for some $\varepsilon>0$. Then there exists a neighbourhood $U$ of $Z$, and a Kähler form $\Omega_{U}=\Omega_{0}+\sqrt{-1} \partial \bar{\partial} \psi_{U}$ on $U$ such that

$$
n\left(1-\frac{\varepsilon}{2}\right) \Omega_{U}^{n-1}-\sum_{k=1}^{n-1} c_{k} k \chi^{n-k} \Omega_{U}^{k-1}>0 .
$$

Note that the hypothesis, in particular implies that $\omega_{Z}$ satisfies the cone condition on $Z$. But to construct a Kähler metric on a neighbourhood $U$ of $Z$, we need this slightly stronger condition.

Proof. Firstly, by compactness of $Z$ we can assume that

$$
\left(1-\frac{\varepsilon}{2}-\delta_{Z}\right) \omega_{Z}^{m}-\sum_{j=0}^{m-1} b_{j} \chi^{m-j} \omega_{Z}^{j}>0,
$$

for some $\delta_{Z}>0$.

Let $\left(U_{\alpha},\left\{z_{\alpha}^{i}\right\}\right)$ be finitely many coordinate charts in $M$ that cover $Z$ and such that $Z \cap U_{\alpha}$ is given by $z_{\alpha}^{m+1}=z_{\alpha}^{m+2}=\cdots=0$. Let $\rho_{\alpha}\left(z_{\alpha}^{1}, z_{\alpha}^{2}, \ldots, z_{\alpha}^{m}\right)$ be a partition-of-unity on $Z$ subordinate to $U_{\alpha} \cap Z$. Let $\pi^{\alpha}$ be the projection to the first $m$ coordinates. Let $\eta_{\alpha}\left(z_{\alpha}^{m+1}, \ldots\right)$ be a smooth function with compact support in the "vertical part" of $U_{\alpha}$ such that it is identically 1 in a neighbourhood of the origin. Then, $\tilde{\rho}_{\alpha}\left(z_{\alpha}\right)=\eta_{\alpha}\left(\pi^{\alpha}\right)^{*} \rho_{\alpha}$ is a partition-of-unity on a closed subset of $\cup_{\alpha} U_{\alpha}$. (Here, we extend $\tilde{\rho}_{\alpha}$ globally by declaring it as 0 outside $U_{\alpha}$.) Abusing notation slightly, we let

$$
\Omega_{U}=\tilde{\Omega}_{U}+C \sqrt{-1} \partial \bar{\partial} d_{\Omega_{0}}(\cdot, Z)^{2},
$$

where $C \geq 1$ is some large constant and

$$
\tilde{\Omega}_{U}=\Omega_{0}+\sqrt{-1} \partial \bar{\partial}\left(\sum_{\alpha} \tilde{\rho}_{\alpha}\left(\pi^{\alpha}\right)^{*} \psi_{Z}\right) .
$$

We note that while $d_{\Omega_{0}}(\cdot, Z)^{2}$ is only Lipschitz globally, it is smooth near $Z$. For small $U$, the form $\Omega_{U}$ is a Kähler metric. By continuity, it is enough to verify the cone condition on $Z$.

Near an arbitrary point $p \in Z$ choose a coordinate chart $\left(U_{\alpha_{0}}, z^{i}\right)$ containing $p$. Extend the tangential coordinate vector fields $\frac{\partial}{\partial z^{1}}, \ldots, \frac{\partial}{\partial z^{m}}$ using an $\Omega_{0}$-orthonormal frame $e_{m+1}, \ldots, e_{n}$ that are orthogonal to the tangential vector fields. For the remainder of this proof, all constants are uniform in $p$ (but depend on the finitely many fixed coordinate charts) and all forms are evaluated at $p$.

Note that

$$
\Omega_{U}(\xi, \bar{\xi}) \geq \tilde{\Omega}_{U}(\xi, \bar{\xi})=\omega_{Z}(\xi, \bar{\xi})
$$


for all tangential directions $\xi$. Moreover, $\sqrt{-1} \partial \bar{\partial} d_{\Omega_{0}}(\cdot, Z)^{2} \geq \frac{1}{K} \sum_{i=m+1}^{n} e^{i} \wedge \bar{e}^{i}$ for some $K>1$. Thus, for any given $0<\delta^{\prime}<1$, we may choose $C=C_{\delta^{\prime}}>1$ large enough so that

$$
\Omega_{U} \geq \Omega_{\delta^{\prime}, U}=\left(1-\delta^{\prime}\right)\left(\left(\pi^{\alpha}\right)^{*} \omega_{Z}+\sqrt{C_{\delta^{\prime}}} \sum_{i=m+1}^{n} e^{i} \wedge \bar{e}^{i}\right)
$$

and

$$
\chi \leq \chi_{\delta^{\prime}, U}=\left(1+\delta^{\prime}\right)\left(\left(\pi^{\alpha}\right)^{*} \chi+\left(C_{\delta^{\prime}}\right)^{1 / 3} \sum_{i=m+1}^{n} e^{i} \wedge \bar{e}^{i}\right) .
$$

It is enough to prove that $\Omega_{\delta^{\prime}, U}$ satisfies the $\frac{\epsilon}{2}$-uniform cone condition with respect to $\chi_{\delta^{\prime}, U}$. Now suppose the eigenvalues of $\Omega_{U}$ at $p$ (with respect to $\chi$ ) are given by $\lambda_{1}, \cdots, \lambda_{n}$ (where $\lambda_{1}, \ldots, \lambda_{m}$ correspond to the invariant subspace spanned by the tangential directions). The inequality 4.7 implies that

$$
1-\frac{\varepsilon}{2}-\delta_{Z}>\sum_{j=0}^{m-1} b_{j}\left(\frac{1-\delta^{\prime}}{1+\delta^{\prime}}\right)^{m-j} \frac{j !(m-j) !}{m !} S_{m-j}\left(\frac{1}{\lambda_{1}}, \cdots, \frac{1}{\lambda_{m}}\right) .
$$

Choose $\delta^{\prime}$ small enough (by choosing a large $C_{\delta^{\prime}}$ ) so that

$$
1-\frac{\varepsilon}{2}-\frac{\delta_{Z}}{2}>\sum_{j=0}^{m-1} b_{j} \frac{j !(m-j) !}{m !} S_{m-j}\left(\frac{1}{\lambda_{1}}, \cdots, \frac{1}{\lambda_{m}}\right)
$$

By choosing an even larger $C_{\delta^{\prime}}$, we may assume that for $k=m+1, \cdots, n, \frac{1}{\lambda_{k}}<\frac{1}{\Lambda}$ for some large $\Lambda$. So we only need to control the terms in $S_{n-k ; i}(1 / \lambda)$ that have no $\lambda_{k}$ for $k \geq m+1$. In particular, we only need to worry about the case when $1 \leq n-k \leq m$ or equivalently $n-m \leq k \leq n-1$. That is, it is enough to prove that

$$
\sum_{k=n-m}^{n-1} \frac{c_{k}}{\left(\begin{array}{l}
n \\
k
\end{array}\right)} S_{n-k}\left(\frac{1}{\lambda_{1}}, \cdots, \frac{1}{\lambda_{m}}\right)<1-\frac{\varepsilon}{2}-\frac{\delta_{Z}}{2} .
$$

But this is precisely (4.9).

Proof of Theorem 1.3. The implication $(1) \Longleftrightarrow(2)$ is proved in Lemma 2.1 and $(2) \Longrightarrow(3)$ is trivial, and hence we only focus on $(3) \Longrightarrow(2)$. We proceed by induction on the dimension of $M$. The theorem is certainly true for $n=1$. Suppose the theorem is true for dimensions less than $n$. The key in making the inductive step work is the following observation. 
Lemma 4.3. Let $Z \subset M$ be a smooth sub-variety of dimension $m<n$. Assuming (3) in the statement Theorem 1.3, there exists a Kähler metric $\omega_{Z}=\left.\Omega_{0}\right|_{Z}+\sqrt{-1} \partial \bar{\partial} \psi_{Z}$ on $Z$ such that

$$
\left(1-\frac{\varepsilon}{2}\right) \omega_{Z}^{m}-\sum_{j=0}^{m-1} b_{j} \chi^{m-j} \omega_{Z}^{j}>0 .
$$

As a consequence, by Lemma 4.2, there exists a neighbourhood $U$ of $Z$ and a Kähler metric $\Omega_{U}=\Omega_{0}+\sqrt{-1} \partial \bar{\partial} \psi_{U}$ on $U$ such that

$$
n\left(1-\frac{\varepsilon}{2}\right) \Omega_{U}^{n-1}-\sum_{k=1}^{n-1} c_{k} k \chi^{n-k} \Omega_{U}^{k-1}>0
$$

Proof. When there is no scope for confusion, we continue to denote $\left.\Omega_{0}\right|_{Z}$ and $\left.\chi\right|_{Z}$ by $\Omega_{0}$ and $\chi$ respectively. Let $a_{Z}$ such that

$$
a_{Z} \int_{Z} \chi^{m}=\int_{Z}\left(\begin{array}{c}
n \\
m
\end{array}\right) \Omega_{0}^{m}-\sum_{j=0}^{m-1} c_{j+n-m}\left(\begin{array}{c}
j+n-m \\
n-m
\end{array}\right) \int_{Z} \chi^{m-j} \Omega_{0}^{j} .
$$

Then by condition $(3), a_{Z}>0$. In fact,

$$
a_{Z}>\varepsilon_{Z}:=\varepsilon\left(\begin{array}{c}
n \\
m
\end{array}\right) \frac{\int_{Z} \Omega_{0}^{m}}{\int_{Z} \chi^{m}} .
$$

For $\omega \in\left[\left.\Omega_{0}\right|_{Z}\right]$, with $b_{j}$ given by(4.6), consider the equation

$$
\left(1-\frac{\varepsilon}{2}\right) \omega^{m}=\sum_{j=1}^{m-1} b_{j} \chi^{m-j} \omega^{j}+f \chi^{m}
$$

where

$$
f=\frac{a_{Z}+c_{n-m}-\varepsilon_{Z} / 2}{\left(\begin{array}{c}
n \\
m
\end{array}\right)}>0 .
$$

Then clearly both sides of the equation integrate out to the same quantity.

Claim. For any sub-variety $V \subset Z$ of co-dimension $p$, we have

$$
\int_{V}\left(\left(\begin{array}{c}
m \\
p
\end{array}\right) \Omega_{0}^{m-p}-\sum_{j=p}^{m-1} b_{j}\left(\begin{array}{c}
j \\
p
\end{array}\right) \chi^{m-j} \Omega_{0}^{j-p}>\varepsilon \int_{V}\left(\begin{array}{c}
m \\
p
\end{array}\right) \Omega_{0}^{m-p}\right.
$$

where $\varepsilon$ is the same as in condition (3).

Proof of the Claim. Since $V$ is of co-dimension $p+n-m$ in $M$, by condition (3) we have

$$
\int_{V}\left(\Omega_{0}^{m-p}-\sum_{k=p+n-m}^{n-1} c_{k} \frac{\left(\begin{array}{c}
k \\
p+n-m
\end{array}\right)}{\left(\begin{array}{c}
n \\
p+n-m
\end{array}\right)} \chi^{n-k} \Omega_{0}^{k-(p+n-m)}\right)>\varepsilon \int_{V} \Omega_{0}^{m-p} .
$$


But then we compute that

$$
\begin{aligned}
& \int_{V} \Omega_{0}^{m-p}-\sum_{j=p}^{m-1} b_{j} \frac{\left(\begin{array}{c}
j \\
p
\end{array}\right)}{\left(\begin{array}{c}
m \\
p
\end{array}\right)} \chi^{m-j} \Omega_{0}^{j-p}=\int_{V} \Omega_{0}^{m-p}-\sum_{j=p}^{m-1} c_{j+n-m} \frac{\left(\begin{array}{c}
j \\
p
\end{array}\right)\left(\begin{array}{c}
j+n-m \\
n-m
\end{array}\right)}{\left(\begin{array}{c}
m \\
p
\end{array}\right)\left(\begin{array}{c}
n \\
m
\end{array}\right)} \chi^{m-j} \Omega_{0}^{j-p}
\end{aligned}
$$

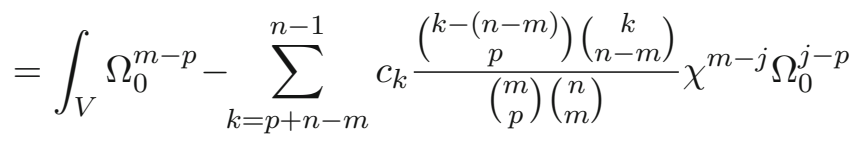

$$
\begin{aligned}
& =\int_{V} \Omega_{0}^{m-p}-\sum_{k=p+n-m}^{n-1} c_{k} \frac{\left(\begin{array}{c}
k \\
p+n-m
\end{array}\right)}{\left(\begin{array}{c}
n \\
p+n-m
\end{array}\right)} \chi^{m-j} \Omega_{0}^{j-p} \\
& \geq \varepsilon \int_{V} \Omega_{0}^{m-p} .
\end{aligned}
$$

From the claim it follows that for any sub-variety $V \subset Z$ of co-dimension $p$ in $Z$, we have

$$
\int_{V}\left(\left(\begin{array}{c}
m \\
p
\end{array}\right) \Omega_{0}^{m-p}-\left(1-\frac{\varepsilon}{2}\right)^{-1} \sum_{j=p}^{m-1} b_{j}\left(\begin{array}{l}
j \\
p
\end{array}\right) \chi^{m-j} \Omega_{0}^{j-p}>\frac{\varepsilon}{2-\varepsilon} \int_{V}\left(\begin{array}{c}
m \\
p
\end{array}\right) \Omega_{0}^{m-p} .\right.
$$

Then by the induction hypothesis and Theorem 2.1 there exists a metric $\omega_{Z} \in\left[\left.\Omega_{0}\right|_{Z}\right]$ solving (4.10). In particular, since $a_{Z}-\varepsilon_{Z} / 2>0$, we have that

$$
\left(1-\frac{\varepsilon}{2}\right) \omega_{Z}^{m}-\sum_{j=0}^{m-1} b_{j} \chi^{m-j} \omega_{Z}^{j}>0
$$

We now return to the proof of Theorem 1.3. We use a continuity method. For $t \geq 0$, consider the following family of equations depending on $t$.

$$
\Omega_{t}^{n}=\sum_{k=1}^{n-1} c_{k} \chi^{n-k} \Omega_{t}^{k}+a_{t} \chi^{n}+f \chi^{n}
$$

where $\Omega_{t} \in(1+t)\left[\Omega_{0}\right]$. Note that $a_{t} \stackrel{t \rightarrow 0^{+}}{\longrightarrow} 0$, and hence we want to solve the above equation at $t=0$. For $t>>1, \hat{\Omega}_{t}=(1+t) \Omega_{0}$ satisfies the cone condition

$$
n \hat{\Omega}_{t}^{n-1}-\sum_{k=1}^{n-1} c_{k} k \chi^{n-k} \hat{\Omega}_{t}^{k-1}>0
$$

By Theorem 2.1, there exists a solution to (4.11) for $t>>1$. In particular, if we let $I=\{t \in[0, \infty) \mid(4.11)$ has a solution $\}$, then $I$ is non-empty. By Lemma 2.1, the cone condition is preserved and hence the linearisation of Equation 4.11 is elliptic. The infinite-dimensional implicit function theorem easily implies that $I$ is open. We need to show that the set is closed. Moreover, by the nature of the cone condition, if $t \in I$, then $t^{\prime} \in I$ for all $t^{\prime}>t$. Let $t_{0}=\inf I$. It is enough to prove that $t_{0} \in I$. 
Replacing $\Omega_{0}$ by $\left(1+t_{0}\right) \Omega_{0}$, without loss of generality, one can assume that $t_{0}=0$, i.e., we have a solution to $(4.11)$ for all $t>0$. The strategy is to produce a $G$-invariant Kähler form $\hat{\Omega}_{0} \in\left[\Omega_{0}\right]$ which satisfies the cone condition. It is actually enough to produce some form satisfying the cone condition. Indeed, by means of averaging over $G$ (with respect to the Haar measure), we get an invariant form. Since the cone condition is convex, it continues to satisfy the cone condition.

Let $Y \subset M$ be a $G$-invariant divisor that contains a Kähler metric $\chi_{Y}$ in its cohomology class. Such a divisor exists by hypothesis of $G$-compatibility. By Theorem 3.1, there exists a $G$-invariant current $\Theta \in\left[\Omega_{0}\right]$ such that $\Theta \geq \beta_{Y}[Y]$ for some $\beta_{Y}>0$, and $\Theta$ satisfies the degenerate cone condition in the sense of Definition 1 . Let $\Xi=\Theta-\frac{\beta_{Y}}{2}[Y]+\frac{\beta_{Y}}{2} \chi_{Y} \in\left[\Omega_{0}\right]$. Then exists an $\varepsilon_{4.1}>0$ such that $T:=\left(1-\varepsilon_{4.1}\right) \Xi$ also satisfies the degenerate cone condition on $M \backslash Y$. Indeed, this statement is easily deduced (going back to $\Omega_{t}$ whose limit is $\Theta$ ) from Lemma 3.2.

With the same notation as Proposition 4.1 (with $\beta=\left(1-\varepsilon_{4.1}\right) \beta_{Y} / 2$ ), let $c<c_{4.1}$, $E_{c}(T)=\{x \in M \mid \nu(T, x) \geq c\}$ and $Z=E_{c}(T) \cup Y$. Clearly, $Z$ is $G$-invariant. By Proposition 4.1, the following is enough.

LEMma 4.4. There exists a neighbourhood $U$ of $Z$ and a Kähler metric $\Omega_{U}=\Omega_{0}+$ $\sqrt{-1} \partial \bar{\partial} \psi_{U}$ such that

$$
n \Omega_{U}^{n-1}-\sum_{k=1}^{n-1} c_{k} k \chi^{n-k} \Omega_{U}^{k-1}>0 .
$$

Proof. If $Z$ is smooth, then we are done by applying Lemma 4.3 to each connected component. In general, $Z$ need not be smooth. Using a canonical resolution of singularities (as in [BM99, KOL07]) there exists a $\pi: \tilde{M}_{r} \stackrel{\pi_{r}}{\longrightarrow} \tilde{M}_{r-1} \ldots \stackrel{\pi_{1}}{\longrightarrow} M_{0}=M$ obtained by successive blowups along smooth $G$-invariant (with respect to the lifted action) centres such that the proper transform $\tilde{Z}_{r}$ of $Z$ is smooth. Note that the induction hypothesis is met for every $\tilde{M}_{i}$. Firstly, we argue that it is enough to assume that $r=1$. In the general case, Lemma 4.5 shows that the numerical condition is satisfied on each $\tilde{M}_{k}$ (and hence in particular, also on $\tilde{Z}_{r}$ and each of the centres $\pi_{k}\left(\tilde{E}_{k}\right)$, where $\tilde{E}_{k}$ are the exceptional divisors). By the discussion below, one starts by constructing a Kähler metric on a neighbourhood $\tilde{U}_{r}$ of $\tilde{Z}_{r}$ in $\tilde{M}_{r}$ satisfying the cone condition. We then proceed recursively. Having constructed a Kähler metric in the nieghbourhood $\tilde{U}_{k}$ of $\tilde{Z}_{k}$, one takes the push forward of the restriction of the metric on $\pi_{k}\left(\tilde{U}_{k} \backslash \tilde{E}_{k}\right)$, and then again by the discussion below, one glues this metric to a metric in the neighbourhood of $\pi_{k}\left(\tilde{E}_{k}\right)$ to obtain a Kähler metric satisfying the cone condition in the neighbourhood of $\tilde{Z}_{k-1}$. Eventually, we obtain a Kähler metric satisfying the cone condition in a neighbourhood of $Z$.

In view of the remarks above, we now assume that $r=1$, that is, $E:=\pi(\tilde{E})$ consists of only one smooth subvariety. It is a standard fact that $\left[\pi^{*} \Omega_{0}\right]-t[\tilde{E}]$ is a $G$-invariant class on $\tilde{M}$ for all $0<t<<1$. In fact, if $h$ is a hermitian metric on $[\tilde{E}]$, 
then for some $C>>1, \tilde{\Omega}_{0}:=\pi^{*} \Omega_{0}+C^{-1} \sqrt{-1} \partial \bar{\partial} \log h$ is a Kähler metric on $\tilde{M}$. For $A>>1$, we let

$$
\left\{\begin{array}{l}
\tilde{\Omega}_{s}=(1+A s) \pi^{*} \Omega_{0}+A s^{2} \tilde{\Omega}_{0} \\
\tilde{\chi}_{s}:=\pi^{*} \chi+s^{2} \tilde{\Omega}_{0} .
\end{array}\right.
$$

To apply our induction hypothesis, we need the following Lemma.

Lemma 4.5. There exists an $A>>1$, such that for all $s<<1$, and all $G$-invariant subvarieties $\tilde{V} \subset \tilde{M}$ of co-dimension $p$, we have

$$
\int_{\tilde{V}}\left(\left(\begin{array}{l}
n \\
p
\end{array}\right) \tilde{\Omega}_{s}^{n-p}-\sum_{k=p}^{n-1} c_{k}\left(\begin{array}{l}
k \\
p
\end{array}\right) \tilde{\chi}_{s}^{n-k} \tilde{\Omega}_{s}^{k-p}\right) \geq \frac{\varepsilon}{4}\left(\begin{array}{l}
n \\
p
\end{array}\right) \int_{\tilde{V}} \tilde{\Omega}_{s}^{n-p} .
$$

We defer the proof of the Lemma, and first use it to complete the proof of Lemma 4.4. By the above Lemma and Lemma 4.3, for all $s<<1$, there exists a neighbourhood $\tilde{W}_{1}$ of $\tilde{Z}$ and a Kähler metric $\tilde{\Omega}_{\tilde{W}_{1}}=\tilde{\Omega}_{s}+\sqrt{-1} \partial \bar{\partial} \tilde{\psi}_{\tilde{W}_{1}}$ satisfying

$$
n\left(1-\frac{\varepsilon}{7}\right) \tilde{\Omega}_{\tilde{W}_{1}}^{n-1}-\sum_{k=1}^{n-1} c_{k} k \tilde{\chi}_{s}^{n-k} \tilde{\Omega}_{\tilde{W}_{1}}^{k-1}>0 .
$$

Similarly, there exists a neighbourhood $\tilde{W}_{2}$ of $\tilde{E}$ and a Kähler metric $\tilde{\Omega}_{\tilde{W}_{2}}=\tilde{\Omega}_{s}+$ $\sqrt{-1} \partial \bar{\partial} \tilde{\psi}_{\tilde{W}_{2}}$ satisfying

$$
n\left(1-\frac{\varepsilon}{7}\right) \tilde{\Omega}_{\tilde{W}_{2}}^{n-1}-\sum_{k=1}^{n-1} c_{k} k \tilde{\chi}_{s}^{n-k} \tilde{\Omega}_{\tilde{W}_{2}}^{k-1}>0 .
$$

As in [CHEN21], we can glue (appropriate modifications of) these metrics to get a Kähler metric $\tilde{\Omega}_{\tilde{U}}=\tilde{\Omega}_{s}+\sqrt{-1} \partial \bar{\partial} \tilde{\psi}_{\tilde{U}}$ on a neighbourhood $\tilde{U}$ of $\tilde{Z} \cup E$ satisfying

$$
n\left(1-\frac{\varepsilon}{8}\right) \tilde{\Omega}_{\tilde{U}}^{n-1}-\sum_{k=1}^{n-1} c_{k} k \tilde{\chi}_{s}^{n-k} \tilde{\Omega}_{\tilde{U}}^{k-1}>0 .
$$

So on $W_{1}:=\pi(\tilde{U} \backslash \tilde{E})$ we have that

$$
n\left(1-\frac{\varepsilon}{8}\right)\left(\pi^{-1}\right)^{*} \tilde{\Omega}_{\tilde{U}}^{n-1}-\sum_{k=1}^{n-1} c_{k} k \chi^{n-k}\left(\pi^{-1}\right)^{*} \tilde{\Omega}_{\tilde{U}}^{k-1}>0 .
$$

Note that $\psi_{W_{1}}:=\tilde{\psi}_{\tilde{U}} \circ \pi^{-1}$ is a bounded function, and moreover,

$$
\left(\pi^{-1}\right)^{*} \tilde{\Omega}_{\tilde{U}}=\Omega_{0}+A s\left(\Omega_{0}+s\left(\pi^{-1}\right)^{*} \tilde{\Omega}_{0}\right)+\sqrt{-1} \partial \bar{\partial} \psi_{W_{1}} .
$$

Now let

$$
\Omega_{W_{1}}:=\frac{\left(\pi^{-1}\right)^{*} \tilde{\Omega}_{\tilde{U}}}{1+A s+A s^{2}}=\Omega_{0}+\sqrt{-1} \partial \bar{\partial}\left(\frac{A C^{-1} s^{2} \pi_{*} \log |\tau|_{h}^{2}+\psi_{W_{1}}}{1+A s+A s^{2}}\right),
$$


where $\tau$ is the defining section of $\tilde{E}$. We claim that if $s$ is chosen sufficiently small, then $\Omega_{W_{1}}$ also satisfies the cone condition. This has to be done a bit carefully since $\Omega_{W_{1}}$ also depends on $s$. But we notice that

$$
\begin{aligned}
(1 & \left.+A s+A s^{2}\right)^{n-1}\left(n\left(1-\frac{\varepsilon}{16}\right) \Omega_{W_{1}}^{n-1}-\sum_{k=1}^{n-1} c_{k} k \chi^{n-k} \Omega_{W_{1}}^{k-1}\right)=n\left(1-\frac{\varepsilon}{8}\right)\left(\pi^{-1}\right)^{*} \tilde{\Omega}_{\tilde{U}}^{n-1} \\
& -\sum_{k=1}^{n-1} c_{k} k \chi^{n-k}\left(\pi^{-1}\right)^{*} \tilde{\Omega}_{\tilde{U}}^{k-1}+\frac{\varepsilon}{16}\left(\pi^{-1}\right)^{*} \tilde{\Omega}_{\tilde{U}}^{n-1} \\
& -\sum_{k=1}^{n-1} c_{k} k\left[\left(1+A s+A s^{2}\right)^{n-k}-1\right] \chi^{n-k}\left(\pi^{-1}\right)^{*} \tilde{\Omega}_{\tilde{U}}^{k-1} .
\end{aligned}
$$

Now if we choose (and fix) an $s<<1$ such that for all $k=1, \cdots, n-1,(1+A s+$ $\left.A s^{2}\right)^{n-k}-1 \leq \frac{\varepsilon}{16}$, then we have that

$$
n\left(1-\frac{\varepsilon}{16}\right) \Omega_{W_{1}}^{n-1}-\sum_{k=1}^{n-1} c_{k} k \chi^{n-k} \Omega_{W_{1}}^{k-1}>0 .
$$

Since $\pi(\tilde{E})$ is smooth, again by induction hypothesis, we obtain a neighbourhood $W_{2}$ of $\pi(\tilde{E})$ and a Kähler metric $\Omega_{W_{2}}=\Omega_{0}+\sqrt{-1} \partial \bar{\partial} \psi_{W_{2}}$ satisfying the cone condition

$$
n\left(1-\frac{\varepsilon}{16}\right) \Omega_{W_{2}}^{n-1}-\sum_{k=1}^{n-1} c_{k} k \chi^{n-k}\left(\pi^{-1}\right)^{*} \Omega_{W_{2}}^{k-1}>0 .
$$

For a large constant $B$, we let

$$
\psi_{U}:=\operatorname{maxx}\left(\frac{A C^{-1} s^{2} \pi_{*} \log |\tau|_{h}^{2}+\psi_{W_{1}}}{1+A s+A s^{2}}+B, \psi_{W_{2}}\right)
$$

on $U=W_{1} \cup W_{2}$. Here mãx is the regularized maximum. The resulting metric $\Omega_{U}:=\Omega_{0}+\sqrt{-1} \partial \bar{\partial} \psi_{U}$ satisfies the required cone condition completing the proof of Lemma 4.4, and hence of Theorem 1.3, subject to proving Lemma 4.5.

We end the section by proving Lemma 4.5 .

Proof of Lemma 4.5. Note that $V=\pi(\tilde{V})$ will also be $G$-equivariant, and so by our hypothesis,

$$
\begin{aligned}
& \int_{\tilde{V}}\left(\left(\begin{array}{l}
n \\
p
\end{array}\right)(1-\varepsilon)(1+A s)^{n-p} \pi^{*} \Omega_{0}^{n-p}\right. \\
& \left.-\sum_{k=p}^{n-1} c_{k}\left(\begin{array}{l}
k \\
p
\end{array}\right)\left((1+A s) \pi^{*} \chi\right)^{n-k}\left((1+A s) \pi^{*} \Omega_{0}\right)^{k-p}\right) \\
& =(1+A s)^{n-p} \int_{V}\left(\left(\begin{array}{l}
n \\
p
\end{array}\right)(1-\varepsilon) \Omega_{0}^{n-p}-\sum_{k=p}^{n-1} c_{k}\left(\begin{array}{l}
k \\
p
\end{array}\right) \chi^{n-k} \Omega_{0}^{k-p}\right) \geq 0 .
\end{aligned}
$$


Hence it suffices to prove that

$$
\begin{aligned}
\int_{\tilde{V}}\left(\left(\begin{array}{c}
n \\
p
\end{array}\right)\left(1-\frac{\varepsilon}{4}\right) \tilde{\Omega}_{s}^{n-p}-\sum_{k=p}^{n-1} c_{k}\left(\begin{array}{l}
k \\
p
\end{array}\right) \tilde{\chi}_{s}^{n-k} \tilde{\Omega}_{s}^{k-p}\right) \\
\geq \int_{\tilde{V}}\left(\left(\begin{array}{c}
n \\
p
\end{array}\right)\left(1-\frac{\varepsilon}{2}\right)(1+A s)^{n-p} \pi^{*} \Omega_{0}^{n-p}\right. \\
\left.\quad-\sum_{k=p}^{n-1} c_{k}\left(\begin{array}{l}
k \\
p
\end{array}\right)\left((1+A s) \pi^{*} \chi\right)^{n-k}\left((1+A s) \pi^{*} \Omega_{0}\right)^{k-p}\right) .
\end{aligned}
$$

Since the integrals are only dependent on the cohomology classes, exactly as in [CHEN21], we replace $\Omega_{0}$ by a more suitable metric to carry out the above estimate. By assumption $E=\pi(\tilde{E})$ is a smooth complex sub-variety of dimension $q$, and hence by Lemma 4.2 there exists a neighbourhood $U$ of $E$ and a Kähler form $\Omega_{U}=\Omega_{0}+\sqrt{-1} \partial \bar{\partial} \psi_{U}$ such that

$$
n\left(1-\frac{3 \varepsilon}{4}\right) \Omega_{U}^{n-1}-\sum_{k=1}^{n-1} c_{k} k \chi^{n-k} \Omega_{U}^{k-1}>0 .
$$

Note that for any $q$,

$$
\left(\begin{array}{l}
n \\
q
\end{array}\right)\left(1-\frac{3 \varepsilon}{4}\right) \Omega_{U}^{n-q}-\sum_{k=q}^{n-1} c_{k}\left(\begin{array}{l}
k \\
q
\end{array}\right) \chi^{n-k} \Omega_{U}^{k-q}>0 .
$$

On $\tilde{M} \backslash \tilde{E}, \tilde{\Omega}_{0}=\pi^{*} \Omega_{0}+C^{-1} \sqrt{-1} \partial \bar{\partial} \log |\tau|_{h}^{2}$, where $\tau$ is the defining section of $\tilde{E}$. We then let $\psi_{1}$ be the regularised maximum of $\psi_{U}$ and $C^{-1} \log |\tau|_{h}^{2}+C^{\prime}$ for some $C^{\prime}>>1$. Then $\Omega_{1}=\Omega_{0}+\sqrt{-1} \partial \bar{\partial} \psi_{1}$ is Kähler on $M$, and $\left.\Omega_{1}\right|_{U^{\prime}}=\left.\Omega_{U}\right|_{U^{\prime}}$ for some $U^{\prime} \subset \subset U$. We then let

$$
\tilde{\Omega}_{1, s}:=(1+A s) \pi^{*} \Omega_{1}+A s^{2} \tilde{\Omega}_{0}
$$

It is enough to prove the following:

$$
\begin{aligned}
& \left(\begin{array}{l}
n \\
p
\end{array}\right)\left(1-\frac{\varepsilon}{4}\right)\left(\tilde{\Omega}_{1, s}^{n-p}-(1+A s)^{n-p} \pi^{*} \Omega_{1}^{n-p}\right) \\
& \geq \sum_{k=p}^{n-1} c_{k}\left(\begin{array}{l}
k \\
p
\end{array}\right)\left(\tilde{\chi}_{s}^{n-k} \tilde{\Omega}_{1, s}^{k-p}-\left((1+A s) \pi^{*} \chi\right)^{n-k}\left((1+A s) \pi^{*} \Omega_{1}\right)^{k-p}\right)
\end{aligned}
$$

We first focus on points $x \in \pi^{-1}\left(U^{\prime}\right)$. Note that in this region $\Omega_{1}$ satisfies (4.12). Also for calculations in the region $\pi^{-1}\left(U^{\prime}\right)$, it is more convenient to work with $\tilde{\chi}_{1, s}:=$ $(1+A s) \pi^{*} \chi+s^{2} \tilde{\Omega}_{0}$. Since $\tilde{\chi}_{1, s} \geq \tilde{\chi}_{s}$, it suffices to prove that for all $x \in \pi^{-1}\left(U^{\prime}\right)$, 


$$
\begin{aligned}
& \left(\begin{array}{c}
n \\
p
\end{array}\right)\left(1-\frac{\varepsilon}{4}\right)\left(\tilde{\Omega}_{1, s}^{n-p}-(1+A s)^{n-p} \pi^{*} \Omega_{1}^{n-p}\right) \\
& \geq \sum_{k=p}^{n-1} c_{k}\left(\begin{array}{l}
k \\
p
\end{array}\right)\left(\tilde{\chi}_{1, s}^{n-k} \tilde{\Omega}_{1, s}^{k-p}-\left((1+A s) \pi^{*} \chi\right)^{n-k}\left((1+A s) \pi^{*} \Omega_{1}\right)^{k-p}\right) .
\end{aligned}
$$

We call the term on the left as $L$ and the term on the right as $R$, and expand:

$$
\begin{aligned}
L-R= & \left(\begin{array}{c}
n \\
p
\end{array}\right)\left(1-\frac{\varepsilon}{4}\right) \sum_{q=p+1}^{n}\left(\begin{array}{c}
n-p \\
n-q
\end{array}\right)(1+A s)^{n-q} \pi^{*} \Omega_{1}^{n-q}\left(A s^{2} \tilde{\Omega}_{0}\right)^{q-p} \\
& -\sum_{k=p}^{n-1} \sum_{a=0}^{n-k} \sum_{b=0}^{k-p} c_{k}\left(\begin{array}{c}
k \\
p
\end{array}\right)\left(\begin{array}{c}
n-k \\
a
\end{array}\right)\left(\begin{array}{c}
k-p \\
b
\end{array}\right) A^{k-p-b} \\
& (1+A s)^{a+b}\left(\pi^{*} \chi\right)^{a}\left(\pi^{*} \Omega_{1}\right)^{b}\left(s^{2} \tilde{\Omega}_{0}\right)^{n-p-a-b} \\
& +\sum_{k=p}^{n-1} c_{k}\left(\begin{array}{c}
k \\
p
\end{array}\right)\left((1+A s) \pi^{*} \chi\right)^{n-k}\left((1+A s) \pi^{*} \Omega_{1}\right)^{k-p} \\
= & \left(\begin{array}{c}
n \\
p
\end{array}\right)\left(1-\frac{\varepsilon}{4}\right) \sum_{q=p+1}^{n}\left(\begin{array}{c}
n-p \\
n-q
\end{array}\right)(1+A s)^{n-q} \pi^{*} \Omega_{1}^{n-q}\left(A s^{2} \tilde{\Omega}_{0}\right)^{q-p}-S_{0}-S_{1} \\
& +\sum_{k=p}^{n-1} c_{k}\left(\begin{array}{c}
k \\
p
\end{array}\right)\left((1+A s) \pi^{*} \chi\right)^{n-k}\left((1+A s) \pi^{*} \Omega_{1}\right)^{k-p},
\end{aligned}
$$

where $S_{0}$ are the terms in the triple summation with $a=0$ (and hence is a double summation), while $S_{1}$ is the triple summation with $a \geq 1$. By changing variable $b=n-q$, ans switching the order of the summations, we can rewrite

$$
S_{0}=\sum_{q=p+1}^{n}\left(\sum_{k=n-(q-p)}^{n-1} c_{k}\left(\begin{array}{l}
k \\
p
\end{array}\right)\left(\begin{array}{l}
k-p \\
n-q
\end{array}\right) A^{k-n}\right)(1+A s)^{n-q}\left(\pi^{*} \Omega_{1}\right)^{n-q}\left(A s^{2} \tilde{\Omega}_{0}\right)^{q-p} .
$$

Now choose $A>>1$, such that for $q=p+1, \cdots, n$,

$$
\sum_{k=n-(q-p)}^{n-1} c_{k}\left(\begin{array}{l}
k \\
p
\end{array}\right)\left(\begin{array}{l}
k-p \\
n-q
\end{array}\right) A^{k-n} \leq \frac{\varepsilon}{4}\left(\begin{array}{l}
n \\
p
\end{array}\right)\left(\begin{array}{l}
n-p \\
n-q
\end{array}\right) .
$$

Note that this can be done since $k-n \leq-1$. Combining with the above, we then obtain

$$
\begin{aligned}
L-R \geq & \left(\begin{array}{l}
n \\
p
\end{array}\right)\left(1-\frac{\varepsilon}{2}\right) \sum_{q=p+1}^{n}\left(\begin{array}{c}
n-p \\
n-q
\end{array}\right)(1+A s)^{n-q} \pi^{*} \Omega_{1}^{n-q}\left(A s^{2} \tilde{\Omega}_{0}\right)^{q-p}-S_{1} \\
& +\sum_{k=p}^{n-1} c_{k}\left(\begin{array}{l}
k \\
p
\end{array}\right)\left((1+A s) \pi^{*} \chi\right)^{n-k}\left((1+A s) \pi^{*} \Omega_{1}\right)^{k-p} .
\end{aligned}
$$


We now focus on $S_{1}$. We change indices: $b=l-q$ and $a=n-l$. Then

$$
\begin{aligned}
S_{1}= & \sum_{k=p}^{n-1} \sum_{l=k}^{n-1} \sum_{q=l-(k-p)}^{l} c_{k}\left(\begin{array}{l}
k \\
p
\end{array}\right)\left(\begin{array}{c}
n-k \\
n-l
\end{array}\right)\left(\begin{array}{c}
k-p \\
l-q
\end{array}\right) A^{k-l} \\
& (1+A s)^{n-q}\left(\pi^{*} \chi\right)^{n-l}\left(\pi^{*} \Omega_{1}\right)^{l-q}\left(A s^{2} \tilde{\Omega}_{0}\right)^{q-p} \\
= & \sum_{q=p}^{n-1} \sum_{l=q}^{n-1} \sum_{k=l-(q-p)}^{l} c_{k}\left(\begin{array}{c}
k \\
p
\end{array}\right)\left(\begin{array}{c}
n-k \\
n-l
\end{array}\right)\left(\begin{array}{c}
k-p \\
l-q
\end{array}\right) A^{k-l} \\
& (1+A s)^{n-q}\left(\pi^{*} \chi\right)^{n-l}\left(\pi^{*} \Omega_{1}\right)^{l-q}\left(A s^{2} \tilde{\Omega}_{0}\right)^{q-p}
\end{aligned}
$$

Let $Q_{0}$ be the term when $k=l$ in the innermost summation, and $Q_{1}$ be the rest. One can choose $A>>1$ (independent of $s$ ) such that for each $q=p, \cdots, n-1$, and for each $l=q, \cdots, n-1$,

$$
\begin{aligned}
& \sum_{k=l-(q-p)}^{l-1} c_{k}\left(\begin{array}{l}
k \\
p
\end{array}\right)\left(\begin{array}{c}
n-k \\
n-l
\end{array}\right)\left(\begin{array}{c}
k-p \\
n-q
\end{array}\right) A^{k-l}(1+A s)^{n-q}\left(\pi^{*} \chi\right)^{n-l}\left(\pi^{*} \Omega_{1}\right)^{l-q}\left(A s^{2} \tilde{\Omega}_{0}\right)^{q-p} \\
& =A^{-1} \sum_{k=l-(q-p)}^{l-1} c_{k}\left(\begin{array}{c}
k \\
p
\end{array}\right)\left(\begin{array}{c}
n-k \\
n-l
\end{array}\right)\left(\begin{array}{c}
k-p \\
n-q
\end{array}\right) A^{k-l+1} \\
& (1+A s)^{n-q}\left(\pi^{*} \chi\right)^{n-l}\left(\pi^{*} \Omega_{1}\right)^{l-q}\left(A s^{2} \tilde{\Omega}_{0}\right)^{q-p} \\
& \leq \frac{\varepsilon}{4 n^{2}}\left(\begin{array}{c}
n \\
p
\end{array}\right)\left(\begin{array}{c}
n-p \\
n-q
\end{array}\right)(1+A s)^{n-q} \pi^{*} \Omega_{1}^{n-q}\left(A s^{2} \tilde{\Omega}_{0}\right)^{q-p},
\end{aligned}
$$

so that summing over $q$ and $l$, we have that

$$
Q_{1} \leq\left(\begin{array}{l}
n \\
p
\end{array}\right) \frac{\varepsilon}{4} \sum_{q=p+1}^{n}\left(\begin{array}{l}
n-p \\
n-q
\end{array}\right)(1+A s)^{n-q} \pi^{*} \Omega_{1}^{n-q}\left(A s^{2} \tilde{\Omega}_{0}\right)^{q-p}
$$

and hence

$$
\begin{aligned}
L-R \geq & \left(\begin{array}{l}
n \\
p
\end{array}\right)\left(1-\frac{3 \varepsilon}{4}\right) \sum_{q=p+1}^{n}\left(\begin{array}{l}
n-p \\
n-q
\end{array}\right)(1+A s)^{n-q} \pi^{*} \Omega_{1}^{n-q}\left(A s^{2} \tilde{\Omega}_{0}\right)^{q-p}-Q_{0} \\
& +\sum_{l=p}^{n-1} c_{l}\left(\begin{array}{l}
l \\
p
\end{array}\right)\left((1+A s) \pi^{*} \chi\right)^{n-l}\left((1+A s) \pi^{*} \Omega_{1}\right)^{l-p}
\end{aligned}
$$

Note that

$$
\left(\begin{array}{l}
n \\
q
\end{array}\right)\left(\begin{array}{l}
l \\
p
\end{array}\right)\left(\begin{array}{l}
l-p \\
l-q
\end{array}\right)=\left(\begin{array}{l}
n \\
p
\end{array}\right)\left(\begin{array}{l}
n-p \\
n-q
\end{array}\right)\left(\begin{array}{l}
l \\
q
\end{array}\right)
$$


and so

$$
\begin{aligned}
Q_{0} & -\sum_{l=p}^{n-1} c_{k}\left(\begin{array}{l}
l \\
p
\end{array}\right)\left((1+A s) \pi^{*} \chi\right)^{n-l}\left((1+A s) \pi^{*} \Omega_{1}\right)^{l-p} \\
& =\sum_{q=p+1}^{n-1} \sum_{l=q}^{n-1} c_{l}\left(\begin{array}{l}
l \\
p
\end{array}\right)\left(\begin{array}{l}
l-p \\
l-q
\end{array}\right)(1+A s)^{n-q}\left(\pi^{*} \chi\right)^{n-l}\left(\pi^{*} \Omega_{1}\right)^{l-q}\left(A s^{2} \tilde{\Omega}_{0}\right)^{q-p} \\
& \leq \sum_{q=p+1}^{n} \sum_{l=q}^{n-1} c_{l}\left(\begin{array}{l}
l \\
p
\end{array}\right)\left(\begin{array}{l}
l-p \\
l-q
\end{array}\right)(1+A s)^{n-q}\left(\pi^{*} \chi\right)^{n-l}\left(\pi^{*} \Omega_{1}\right)^{l-q}\left(A s^{2} \tilde{\Omega}_{0}\right)^{q-p} \\
& =\sum_{q=p+1}^{n} \frac{\left(\begin{array}{l}
n-p \\
n-q
\end{array}\right)\left(\begin{array}{l}
n \\
p
\end{array}\right)}{\left(\begin{array}{l}
n \\
q
\end{array}\right)}\left(\sum_{l=q}^{n-1} c_{l}\left(\begin{array}{l}
l \\
q
\end{array}\right)\left(\pi^{*} \chi\right)^{n-l}\left(\pi^{*} \Omega_{1}\right)^{l-q}\right)(1+A s)^{n-q}\left(A s^{2} \tilde{\Omega}_{0}\right)^{q-p} \\
& \leq\left(\begin{array}{l}
n \\
p
\end{array}\right)\left(1-\frac{3 \varepsilon}{4}\right) \sum_{q=p+1}^{n}\left(\begin{array}{l}
n-p \\
n-q
\end{array}\right)\left(\pi^{*} \Omega_{1}\right)^{n-q}(1+A s)^{n-q}\left(A s^{2} \tilde{\Omega}_{0}\right)^{q-p} .
\end{aligned}
$$

To recap, for any $x \in \pi^{-1}\left(U^{\prime}\right)$, we have verified that (4.14), and hence (4.13), holds.

To complete the proof, we consider the points in the set $\tilde{M} \backslash \pi^{-1}\left(U^{\prime}\right)$. We now go back to working with $\tilde{\chi}_{s}$ and prove the inequality in (4.13) directly. On this set, $\pi$ is a bi-holomorphism and hence for some uniform $C$ (independent of $s$, so long as $s<1)$,

$$
\begin{aligned}
& C^{-1} \pi^{*} \chi \leq \pi^{*} \Omega_{1} \leq C \pi^{*} \chi \\
& C^{-1} \pi^{*} \chi \leq \tilde{\Omega}_{0} \leq C \pi^{*} \chi .
\end{aligned}
$$

Consider the difference of the two sides in (4.13). The $O(1)$ terms (as $s \rightarrow 0$ ) cancel out, and the leading term (or the $O(s)$ term) is given by $\mathrm{s}$

$$
A s \sum_{k=p}^{n-1} c_{k}(n-k)\left(\begin{array}{l}
k \\
p
\end{array}\right)\left(\pi^{*} \chi\right)^{n-k}\left((1+A s) \pi^{*} \Omega_{1}\right)^{k-p},
$$

which is strictly positive since at least one of $c_{k}$ is strictly positive and others are non-negative. The rest of the terms are $O\left(s^{2}\right)$ (since they contain at least one copy of $s^{2} \tilde{\Omega}_{0}$ ), and hence for all $s<s_{0}$, where $s_{0}$ only depends on the constant $C$ above, the difference of the two sides in (4.13) is positive.

\section{Proof of Theorem 1.1}

In this section, we prove Theorem 1.1. In particular, we assume that the (nonuniform) numerical condition holds, i.e., for any subvariety $V \subset M$,

$$
\int_{V}\left(\left(\begin{array}{l}
n \\
p
\end{array}\right)\left[\Omega_{0}\right]^{n-p}-\sum_{k=p}^{n-1} c_{k}\left(\begin{array}{l}
k \\
p
\end{array}\right)\left[\chi^{n-k}\right] \cdot\left[\Omega_{0}^{k-p}\right]\right)>0 .
$$


We prove Theorem 1.1 by induction on $n=\operatorname{dim}(M)$ just as in the case of Theorem 1.3. For $n=1$, the result is trivial. Assume that it holds for $1,2, \ldots, n-1$. Proposition 4.1 shows that the proof of Theorem 1.3 carries over verbatim provided we prove the following stronger version of Lemma 4.4 .

Proposition 5.1. Let $Z \subset M$ be a closed analytic subset. Assume the (nonuniform) numerical condition (5.1) holds. Then there exists a neighbourhood $U$ of $Z$ and a Kähler metric $\Omega_{U}=\Omega_{0}+\sqrt{-1} \partial \bar{\partial} \psi_{U}$ on $U$ such that

$$
n \Omega_{U}^{n-1}-\sum_{k=1}^{n-1} k c_{k} \chi^{n-k} \Omega_{U}^{k-1}>0 .
$$

To prove Proposition 5.1, we first prove a lemma that is similar to, but stronger than Lemma 4.2 .

LEMMA 5.1. Let $Z \subset M$ be a smooth $m$-dimensional subvariety. Let $\Omega_{0}$ and $\chi$ be Kähler forms on $M$. Suppose there exists a smooth Kähler form $\omega_{Z}=\left.\Omega_{0}\right|_{Z}+$ $\sqrt{-1} \partial \bar{\partial} \psi_{Z}$ on $Z$ such that

$$
\omega_{Z}^{m}-\sum_{j=0}^{m-1} b_{j} \chi^{m-j} \omega_{Z}^{j}>0 .
$$

Then there exists a neighbourhood $U$ of $Z$, a smooth function $\psi_{U}: U \rightarrow \mathbb{R}$ such that the smooth form $\Omega_{U}=\Omega_{0}+\sqrt{-1} \partial \bar{\partial} \psi_{U}$ is Kähler and satisfies

$$
n \Omega_{U}^{n-1}-\sum_{k=1}^{n-1} k c_{k} \chi^{n-k} \Omega_{U}^{k-1}>0
$$

on $U$. In particular, $\Omega_{U}$ satisfies the cone condition on $U$.

Proof. Let $\operatorname{dist}(., Z)$ be the distance (with respect to some Kähler metric on $M$ ) to $Z$ and $\psi^{\prime}$ the extension of $\psi$ (as in the proof of Lemma 4.2) to a neighbourhood of $Z$. Then, for a large enough constant $C, \tilde{\psi}=\psi^{\prime}+C \operatorname{dist}(., Z)^{2}$ is a smooth function in a neighbourhood $U$ of $Z$ satisfying the desired conditions.

Using this lemma, the induction hypothesis, and the proof of Lemma 4.3, we see that the following non-uniform version of Lemma 4.3 holds.

Lemma 5.2. Let $Z \subset M$ be a smooth sub-variety of dimension $m<n$. Assuming the (non-uniform) numerical condition (5.1), there exists a neighbourhood $U$ of $Z$ and a Kähler metric $\Omega_{U}=\Omega_{0}+\sqrt{-1} \partial \bar{\partial} \psi_{U}$ on $U$ such that

$$
n \Omega_{U}^{n-1}-\sum_{k=1}^{n-1} c_{k} k \chi^{n-k} \Omega_{U}^{k-1}>0 .
$$

We only need to observe that the quantity $a_{Z}$ in the proof of Lemma 4.3 is still positive, and that we can apply the same argument with $\varepsilon_{Z}=a_{Z} / 2$. Now we proceed to prove Proposition 5.1. 
5.1 Proof of Proposition 5.1. We induct on the maximal dimension $m<n$ over all irreducible components of $Z$. For $m=0$, we have isolated points, for which the proposition is trivially true. Assume it is true for $0,1,2 \ldots, m-1$. Removing the isolated points of $Z$, without loss of generality, we may assume that the dimension of every irreducible component of $Z$ is $\geq 1$.

As in the proof of Theorem 1.3, we resolve $Z$ to a disjoint union of smooth manifolds $\tilde{Z}=Z_{r}$ via a sequence of $r$ blowups $M_{1}, M_{2} \ldots$ at smooth centres $C_{i} \subset$ $Z_{i}$ where $Z_{i}$ are proper transforms. Let $\pi: \tilde{Z} \rightarrow Z$ be the projection map with $\pi^{-1}\left(Z_{\text {sing }}\right)=E$ as the union of all the exceptional divisors $E_{i}$. There exists an ample line bundle $L$ over $M$. For some large $N$, the bundle $L^{N}$ has a holomorphic section $\mathcal{S}_{Y}$ whose zero locus $Y$ is a smooth connected hypersurface such that $Y_{1}=Y \cap Z$ is a divisor in $Z^{1}$. Let $N$ also be so large that $\tilde{L}_{i}=\pi^{*} L^{N} \otimes\left[-E_{i}\right] \otimes\left[-E_{i-1}\right] \ldots$ is an ample line bundle over $M_{i}$ for all $1 \leq i \leq r$. Let $h$ be a metric on $L$ (over $M$ ) of positive curvature $F_{h}$ and $h_{i}$ be metrics on $\left[-E_{i}\right]$ such that $F_{h_{i}} \geq-\frac{N}{10 r} \pi_{i}^{*} F_{h}$ and is positive when restricted to $E_{i}$. Then $H_{i}=\pi_{i}^{*} h^{N} \otimes \Pi_{j} h_{j}$ is a metric on $\tilde{L}_{i}$ of positive curvature $F_{H_{i}}$. Denote by $\tilde{\Omega}_{0}$ the Kähler form $\pi^{*} \Omega_{0}+C^{-1} F_{H_{r}}$ for a sufficiently large $C$.

Now define $\tilde{\chi}_{s}:=\pi^{*} \chi+s^{5 n} \tilde{\Omega}_{0}$ for $1 \geq s \geq 0$, and

$$
\tilde{a}_{s, s_{0}}=\frac{\int_{\tilde{M}}\left(\tilde{\chi}_{s}^{n}-\tilde{\chi}_{s_{0}}^{n}\right)}{\int_{\tilde{M}} \pi^{*} \chi^{n}} .
$$

Note that $\tilde{\chi}_{s} \leq C_{\chi} \tilde{\Omega}_{0}$ for some $C_{\chi}>0$ independent of $0 \leq s \leq 1$. We fix $\tilde{\varepsilon}$ and $s_{0}<1$ for the remainder of this paper so that $\tilde{\chi}_{s_{0}}$ is Kähler for all $0<s<s_{0}$ and the following hold for all $0<s<s_{0}$ and $0 \leq t \leq 1$.

$$
\begin{array}{r}
200 \tilde{a}_{s, s_{0}}>\frac{f_{m}}{4^{n}}, \text { and } \\
\tilde{\chi}_{s_{0}}-\tilde{\chi}_{\frac{s_{0}}{2}}-\tilde{\varepsilon} N \frac{\pi^{*} F_{h}\left|\pi^{*} \mathcal{S}_{Y}\right|_{h^{N}}^{2}}{\left|\pi^{*} \mathcal{S}_{Y}\right|_{h^{N}}^{2}+t^{2}} \geq 0,
\end{array}
$$

where $f_{m}$ is as in 1.3 .

Our first step is to state and prove another concentration of mass result (like Theorem 3.1). We need the following definitions, which generalise Definition 1 in two directions, namely allowing powers of arbitrary order, and allowing $\chi$ to be possibly only semi-positive.

Definition 2. Let $\Theta$ be a closed, positive $(1,1)$ current and $\chi$ a Kähler form on an $n$-dimensional Kähler manifold. We say that $\Theta$ satisfies the $(\varepsilon, n-p)$-uniform cone

1 Indeed, if $\operatorname{dim}(Z)=1$, then for large $N, Y \cap Z$ is a non-empty collection of points and is hence a divisor in $Z$. If $\operatorname{dim}(Z) \geq 2$, then since $\operatorname{dim}(Y)+\operatorname{dim}(Z)>\operatorname{dim}(M)$ the analytic set $Y \cap Z$ is a union of connected subvarieties by the Fulton-Hansen connectedness theorem. Hence, if we ensure that there exists a $Y$ that intersects every component of $Z$ in at least one smooth point transversally, we are done by Bertini's theorem. 
condition $(0 \leq p \leq n)$ :

$$
\left(\begin{array}{l}
n \\
p
\end{array}\right)(1-\varepsilon) \Theta^{n-p}-\sum_{k=p}^{n-1} c_{k}\left(\begin{array}{l}
k \\
p
\end{array}\right) \chi^{n-k} \Theta^{k-p} \geq 0,
$$

if for any coordinate chart $U$ with $\left.\Theta\right|_{U}=\sqrt{-1} \partial \bar{\partial} \varphi_{U}$, on $U_{\delta}:=\{x \in M \mid B(x, \delta) \subset$ $U\}$ (where $B(x, \delta)$ is a coordinate Euclidean ball of radius $\delta$ centred at $x$ ), we have

$$
\left(\begin{array}{l}
n \\
p
\end{array}\right)(1-\varepsilon)\left(\sqrt{-1} \partial \bar{\partial} \varphi_{U, \delta}\right)^{n-p}(x)-\sum_{k=p}^{n-1} c_{k} k \chi_{0}^{n-k}\left(\sqrt{-1} \partial \bar{\partial} \varphi_{U, \delta}\right)^{k-p}(x) \geq 0,
$$

for any Kähler metric $\chi_{0}$ on $U$ with constant coefficients satisfying $\chi_{0} \leq \chi$ on $B(x, \delta)$. Here $\varphi_{U, \delta}$ are the mollifications of $\varphi_{U}$ as before.

Note that in particular, Definition 1 defines an $(\varepsilon, n-1)$-uniform cone condition.

Definition 3. Let $\Theta$ be a closed, positive $(1,1)$-current and $\chi$ be a semi-positive smooth closed $(1,1)$-form on a Kähler manifold of dimension $n$. We say that $\Theta$ satisfies the $(n-p)$-degenerate strong cone condition

$$
\left(\begin{array}{l}
n \\
p
\end{array}\right) \Theta^{n-p}-\sum_{k=1}^{n} c_{k}\left(\begin{array}{l}
k \\
p
\end{array}\right) \chi^{n-k} \Theta^{k} \geq 0
$$

if there exist positive sequences $\varepsilon_{i}, \mu_{i}, \nu_{i}, \gamma_{i} \rightarrow 0$ and a smooth Kähler form $\tilde{\Omega}_{0}$, such that the closed positive current $\Theta_{i}=\Theta\left(1+\gamma_{i}\right)+\mu_{i} \tilde{\Omega}_{0}$ satisfies the $\left(\varepsilon_{i}, n\right)$-uniform cone condition with respect to the Kähler form $\chi_{i}=\chi+\nu_{i} \tilde{\Omega}_{0}$ for every $i$.

REMARK 5.1. It is easy to see that for a smooth current $\Theta$, Definition 3 is equivalent to the usual pointwise definition. It is also not hard to see that Definition 3 generalises Definition 1.

Recall that $Y \subset M$ is an ample divisor such that $Y$ intersects every irreducible component of $Z$ in a divisor. Define $\tilde{Y}=\pi^{-1} Y \cap \tilde{Z}$ and $\tilde{E}=E \cap \tilde{Z}$. Note that $\tilde{Y}$ and $\tilde{E}$ are divisors in $\tilde{Z}$. In fact, $E$ intersects $\tilde{Z}$ in simple normal crossings. Denote $\pi^{*} \chi$ by $\tilde{\chi}$. Once again, as in equation (4.6), for $m<n$ and $j=0, \cdots, m-1$, we let

$$
b_{j}:=\frac{c_{j+n-m}\left(\begin{array}{c}
j+n-m \\
n-m
\end{array}\right)}{\left(\begin{array}{c}
n \\
m
\end{array}\right)} .
$$

Proposition 5.2. There exists a constant $\beta^{\prime}>0$ and a current $\Theta \in\left[\pi^{*} \Omega_{0}\right]$ on $\tilde{Z}$ such that $\Theta \geq 2 \beta^{\prime}[\tilde{Y}]$ and $\Theta$ satisfies the following $m$-degenerate strong cone condition (in the sense of Definition 3):

$$
\Theta^{m}-\sum_{j=0}^{m-1} b_{j} \pi^{*} \chi^{m-j} \Theta^{j} \geq 0
$$


To this end, we first need the following general lemma regarding the cone condition. This is a stronger (i.e. degenerate) version of Lemma 3.2.

Lemma 5.3. Let $\tilde{Z}$ be an $m$-dimensional Kähler submanifold of an $n$-dimensional Kähler manifold, and for $0 \leq j \leq m-1$, let $b_{j} \geq 0$ such that $\sum_{j} b_{j}>0$. Furthermore, let $\Omega, \chi \geq 0$ be smooth forms such that for all $0 \leq p \leq m-1$, the following point-wise inequality is satisfied at a point $x \in \tilde{Z}$ :

$$
\frac{1}{(1+2 \varepsilon)^{2}} \Omega^{m-p}(x)-\sum_{j=p}^{m-1} \frac{\left(\begin{array}{c}
j \\
p
\end{array}\right)}{\left(\begin{array}{c}
m \\
p
\end{array}\right)} b_{j} \chi^{m-j} \Omega^{j-p}(x) \geq 0 .
$$

Assume that $\chi(x) \leq C_{\chi} \tilde{\Omega}_{0}(x)$ for some $C_{\chi}>0$. Then there exists an $s_{0}^{\prime}=s_{0}^{\prime}\left(C_{\chi}, m\right.$, $\left.b_{j}, \epsilon\right)$ such that for all $s<s_{0}^{\prime}$, the following point-wise inequality holds at $x$ :

$$
\begin{aligned}
& \frac{1}{(1+\varepsilon)^{2}}\left(\Omega(1+10 s)+10 s^{2} \tilde{\Omega}_{0}\right)^{m}(x) \\
& \quad-\sum_{j=0}^{m-1} b_{j}\left(\chi+s^{5 n} \tilde{\Omega}_{0}\right)^{m-j}\left(\Omega(1+10 s)+10 s^{2} \tilde{\Omega}_{0}\right)^{j}(x) \geq 0,
\end{aligned}
$$

Proof. The left hand side of Inequality 5.8 can be written as $P+Q$, where :

$$
\begin{aligned}
P:= & \frac{1}{(1+2 \varepsilon)^{2}}\left(\Omega(1+10 s)+10 s^{2} \tilde{\Omega}_{0}\right)^{m}-\sum_{j=0}^{m-1} b_{j} \chi^{m-j}\left(\Omega(1+10 s)+10 s^{2} \tilde{\Omega}_{0}\right)^{j} \\
Q:= & \left(\frac{1}{(1+\varepsilon)^{2}}-\frac{1}{(1+2 \varepsilon)^{2}}\right)\left(\Omega(1+10 s)+10 s^{2} \tilde{\Omega}_{0}\right)^{m} \\
& -\sum_{j=0}^{m-1} \sum_{h=0}^{m-j-1} b_{j}\left(\begin{array}{c}
m-j \\
h
\end{array}\right) \chi^{h} s^{5 n(m-j-h)} \tilde{\Omega}_{0}^{m-j-h}\left(\Omega(1+10 s)+10 s^{2} \tilde{\Omega}_{0}\right)^{j}
\end{aligned}
$$

We first prove that $P \geq 0$. In the following calculation, by definition $\left(\begin{array}{l}n \\ r\end{array}\right)=0$ when $r>n$.

$$
\begin{aligned}
P= & \sum_{p=0}^{m}\left(10 s^{2}\right)^{p} \tilde{\Omega}_{0}^{p} \frac{1}{(1+2 \varepsilon)^{2}}\left(\begin{array}{c}
m \\
p
\end{array}\right) \Omega^{m-p}(1+10 s)^{m-p} \\
& -\sum_{j=0}^{m-1} b_{j} \chi^{m-j} \sum_{p=0}^{j}\left(10 s^{2}\right)^{p} \tilde{\Omega}_{0}^{p}\left(\begin{array}{c}
j \\
p
\end{array}\right) \Omega^{j-p}(1+10 s)^{j-p} \\
\geq & \sum_{p=0}^{m}\left(10 s^{2}\right)^{p}(1+10 s)^{m-p} \tilde{\Omega}_{0}^{p}\left(\frac{1}{(1+2 \varepsilon)^{2}}\left(\begin{array}{c}
m \\
p
\end{array}\right) \Omega^{m-p}-\sum_{j=0}^{m-1} b_{j} \chi^{m-j}\left(\begin{array}{l}
j \\
p
\end{array}\right) \Omega^{j-p}\right) \\
\geq & 0,
\end{aligned}
$$

using Inequalities 5.7. 
Next, we prove that $Q \geq 0$, thus completing the proof of Lemma 5.3. To begin with, since $\chi \leq C_{\chi} \tilde{\Omega}_{0}$, and $5 m \leq 5 n(m-j-h)$ for all $0 \leq h \leq m-j-1$, assuming that $s<1$ we get the following inequality.

$$
\begin{aligned}
Q \geq & \left(\frac{1}{(1+\varepsilon)^{2}}-\frac{1}{(1+2 \varepsilon)^{2}}\right)\left(\Omega(1+10 s)+10 s^{2} \tilde{\Omega}_{0}\right)^{m} \\
& -\sum_{j=0}^{m-1} \sum_{h=0}^{m-j-1} b_{j}\left(\begin{array}{c}
m-j \\
h
\end{array}\right) C_{\chi}^{h} s^{5 m} \tilde{\Omega}_{0}^{m-j}\left(\Omega(1+10 s)+10 s^{2} \tilde{\Omega}_{0}\right)^{j} .
\end{aligned}
$$

We continue simplifying further to get the following (using $s<1$ ).

$$
\begin{aligned}
\Rightarrow Q \geq & \sum_{j=0}^{m-1}\left(\Omega(1+10 s)+10 s^{2} \tilde{\Omega}_{0}\right)^{j}\left(s^{2} \tilde{\Omega}_{0}\right)^{m-j}\left(\left(\frac{1}{(1+\varepsilon)^{2}}-\frac{1}{(1+2 \varepsilon)^{2}}\right) 10^{m-j}\right. \\
& \left.-\sum_{h=0}^{m-j-1} b_{j}\left(\begin{array}{c}
m-j \\
h
\end{array}\right) C_{\chi}^{h} s^{3 m}\right) \\
\geq & 0
\end{aligned}
$$

for sufficiently small $s$ (depending only on $C_{\chi}, m, b_{j}, \epsilon$ ).

Now we prove Proposition 5.2.

Proof. For $0<t<1$, the Kähler classes $\left[\Omega_{t}\right]:=(1+t)\left[\Omega_{0}\right]$ satisfy the uniform numerical criteria (with the $\varepsilon$ possibly depending on $t$ ). Then by Lemma 4.5 , there exists $A_{t}>0$ and $\frac{s_{0}}{2}>\bar{s}_{t}>0$ such that the classes $\left(1+A_{t} s\right)\left[\pi^{*} \Omega_{t}\right]+A_{t} s^{2}\left[\tilde{\Omega}_{0}\right]$ and $\left[\tilde{\chi}_{s, t}\right]:=\left[\pi^{*} \chi\right]+s^{2}\left[\tilde{\Omega}_{0}\right]$ satisfies the uniform numerical criteria for all $s<\bar{s}_{t}<\frac{s_{0}}{2}<1$. In particular, the uniform numerical criteria are satisfied with $\left[\tilde{\chi}_{s, t}\right]$ replaced by $\left[\tilde{\chi}_{s}\right]$. Now let $s_{t}:=\frac{\bar{s}_{t}\left(1-e^{-t}\right)}{2 A_{t}}$, and $\left[\tilde{\Omega}_{s_{t}}\right]:=\left(1+A_{t} s_{t}\right)\left[\pi^{*} \Omega_{t}\right]+A_{t} s_{t}^{2}\left[\tilde{\Omega}_{0}\right]$. Then $\left[\tilde{\Omega}_{s_{t}}\right]$ and $\left[\tilde{\chi}_{s_{t}}\right]$ satisfy the uniform numerical criteria (where once again the uniformity might depend on $t$ ). Consider

$$
\mathcal{B}_{s_{t}}:=\frac{\int_{\tilde{M}}\left(\left[\tilde{\Omega}_{s_{t}}\right]^{n}-\sum_{j=1}^{n-1} c_{j} \tilde{\chi}_{s_{t}}^{n-j}\left[\tilde{\Omega}_{s_{t}}\right]^{j}-\tilde{\chi}_{s_{0}}^{n}+\tilde{\chi}_{s_{t}}^{n}\right)}{\int_{\tilde{M}} \tilde{\chi}_{s_{t}}^{n}} .
$$

Note that $\mathcal{B}_{s_{t}}$ can be negative but since by assumption $s_{t}<\frac{s_{0}}{2}$ for all $0<t<1$, we see that

$$
\mathcal{B}_{s_{t}} \geq a_{s_{t}, s_{0}}>\frac{f_{m}}{4^{n} 200}
$$

where $f_{m}$ is as in (1.2).

Next, as before, we construct some model forms that concentrate on $\tilde{Y}$. We let

$$
\tilde{\eta}_{t}=\tilde{\chi}_{s_{0}}+\tilde{\varepsilon} \sqrt{-1} \partial \bar{\partial} \log \left(\left|\pi^{*} S\right|_{\pi^{*} h^{N}}^{2}+t^{2}\right) .
$$


We then find a metric $\tilde{\Omega}_{s_{t}} \in\left[\tilde{\Omega}_{s_{t}}\right]$ on $\tilde{M}$ solving

$$
\tilde{\Omega}_{s_{t}}^{n}=\sum_{j=1}^{n-1} c_{j} \tilde{\chi}_{s_{t}}^{n-j} \tilde{\Omega}_{s_{t}}^{j}+f_{t} \tilde{\chi}_{s_{t}}^{n}
$$

where $f_{t}:=\frac{\tilde{\eta}_{t}^{n}}{\tilde{\chi}_{s_{t}}^{n}}-1+\mathcal{B}_{s_{t}}$. Indeed, noting that

$$
\int_{\tilde{M}} \tilde{\Omega}_{s_{t}}^{n} \leq \int_{\tilde{M}} 2^{n}\left((1+t)^{n} \pi^{*} \Omega_{0}^{n}\left(1+A_{t} s_{t}\right)^{n}+A_{t}^{n} s_{t}^{2 n} \tilde{\Omega}_{0}^{n}\right),
$$

and using Inequality 5.11, we see that $f_{t}$ satisfies the conditions in Theorem 1.3 for all $0<t<<1$.

Moreover, since the uniform positivity criteria are satisfied for every $0<t<1$, by Theorem 1.3, there exists a smooth solution of 5.12 for all $0<t<<1$. By Lemma 2.1 we see that the cone condition is met by $\tilde{\Omega}_{s_{t}}$ with respect to $\tilde{\chi}_{s_{t}}$. Choose a positive sequence $\gamma_{i} \rightarrow 0$. There exists a sequence $\delta_{i}>0$ such that the $\left(2 \delta_{i}, n\right)$-uniform cone condition is satisfied by $\tilde{\Omega}_{s_{t}}\left(1+\gamma_{i}\right)$ with respect to $\tilde{\chi}_{s_{t}}$. Lemma 5.3 shows that there exists a positive sequence $\mu_{i}$ converging to 0 such that $\varepsilon_{i}$-cone condition is satisfied by $\tilde{\Omega}_{s_{t}}\left(1+\gamma_{i}\right)\left(1+10 \mu_{i}\right)+10 \mu_{i}^{2} \tilde{\Omega}_{0}$ with respect to $\tilde{\chi}_{s_{t}}+\mu_{i}^{5 n} \tilde{\Omega}_{0}$ for some $\varepsilon_{i}$ depending explicitly on $\delta_{i}$. Denote a weak limit of $\tilde{\Omega}_{s_{t}}$ by $\Theta$. Then arguing just as in the proof of Theorem 3.1, it is easily seen that $\Theta\left(1+\gamma_{i}\right)\left(1+10 \mu_{i}\right)+10 \mu_{i}^{2} \tilde{\Omega}_{0}$ satisfies the $\left(\varepsilon_{i}, m\right)$-uniform cone condition with respect to the Kähler form $\pi^{*} \chi+\mu_{i}^{5 n} \tilde{\Omega}_{0}$ as in Definition 2, and hence $\Theta$ satisfies the $m$-degenerate strong cone condition as in Definition 3.

To complete the proof of the Proposition, it is then enough to prove the following analogue of Lemma 3.1 to prove that any weak limit $\Theta$ of $\left.\tilde{\Omega}_{s_{t}}\right|_{Z}$ satisfies $\Theta \geq 2 \beta^{\prime}[\tilde{Y}]$.

Lemma 5.4. For any $y \in \tilde{Y}$, there exists a neighbourhood $U$ and a constant $\beta_{U}>0$ such that for all $t<<t_{0}$,

$$
\int_{U \cap V_{t}} \tilde{\Omega}_{s_{t}} \wedge \tilde{\chi}_{s_{0}}^{m-1}>\beta_{U}
$$

where as before, $V_{t}=\left\{|S|_{h^{N}}<t\right\}$.

The proof proceeds exactly as in Lemma 3.1. The key observation is that $\tilde{\chi}_{s_{0}}$ is Kähler throughout $\tilde{Z}$, and hence the argument in proof of Lemma 2.1 in [DP01] implies that for each $y \in \tilde{Y}$ there exists a neighbourhood $U$ and a constant $\beta(U)$ such that

$$
\int_{U \cap V_{t}} \tilde{\eta}_{t} \wedge \tilde{\chi}_{s_{0}}^{m-1}>\beta(U)
$$


Now, since $\tilde{\Omega}_{s_{t}}$ satisfies

$$
n \tilde{\Omega}_{s_{t}}^{n-1}-\sum_{k=1}^{n-1} k c_{k} \tilde{\chi}_{s_{t}}^{n-k} \tilde{\Omega}_{s_{t}}^{k-1} \geq 0
$$

we see that

$$
\tilde{\Omega}_{s_{t}}^{m}-\sum_{j=0}^{m-1} b_{j} \tilde{\chi}_{s_{t}}^{m-j} \tilde{\Omega}_{s_{t}}^{j} \geq 0
$$

when restricted to $\tilde{Z}$.

Akin to the proof of Proposition 5.2 , denote by $\tilde{\Omega}_{s}$ the form $\pi^{*} \Omega_{0}(1+10 s)+$ $10 s^{2} \tilde{\Omega}_{0}$. We now use Proposition 5.2 to prove a similar proposition (with more positivity in some sense).

Proposition 5.3. There exist constants $\beta^{\prime \prime}>0, K>1, \epsilon>0$ and $s_{0}^{\prime}<s_{0} / 2$ such that for all $s<s_{0}^{\prime}$, there exists a Kähler current $T_{s} \in\left[\tilde{\Omega}_{s}\right]$ on $\tilde{Z}$ with the following properties.

(1) $T_{s}^{\prime}=T_{s}-250 \epsilon \tilde{\Omega}_{0} \geq \beta^{\prime}[\tilde{Y}]+\beta^{\prime \prime}[\tilde{E}]+\frac{\tilde{\Omega}_{0}}{K}$. In particular, $T_{s}$ and $T_{s}^{\prime}$ have Lelong numbers at least $\min \left(\beta^{\prime}, \beta^{\prime \prime}\right)$ along $\tilde{Y} \cup \tilde{E}$.

(2) For all $c>0$, the Lelong sublevel set $E_{c}\left(T_{s}\right) \subset E_{\frac{c}{3}}(\Theta) \cup \tilde{Y} \cup \tilde{E}$.

(3) $T_{s}^{\prime}$ satisfies the following degenerate cone condition (in the sense of Definition 1) away from $\tilde{Y} \cup \tilde{E}$ :

$$
\frac{1}{(1+\epsilon)^{2}}\left(T_{s}^{\prime}\right)^{m}-\sum_{j=0}^{m-1} b_{j} \tilde{\chi}_{s}^{m-j}\left(T_{s}^{\prime}\right)^{j} \geq 0
$$

Proof of Proposition 5.3. Let $s_{E}$ be an induced section over $\tilde{M}$ of $[E]$ defining $E$. For small enough $\beta^{\prime \prime}$, we can easily see that the current $T_{0}=\Theta-\beta^{\prime} \sqrt{-1} \partial \bar{\partial} \ln \left|\pi^{*} \mathcal{S}_{Y}\right|_{h^{N}}^{2}+$ $\beta^{\prime \prime} \sqrt{-1} \partial \bar{\partial} \ln \left|s_{E}\right|_{\otimes h_{i}^{-1}}^{2}$ is Kähler throughout $\tilde{Z}$ and satisfies $T_{0}^{\prime}=T_{0}-250 \epsilon_{0} \tilde{\Omega}_{0} \geq$ $\beta^{\prime}[\tilde{Y}]+\beta^{\prime \prime}[\tilde{E}]+\frac{\tilde{\Omega}_{0}}{K}$ for constants $\epsilon_{0}>0$ and $K>1$. In fact, going back to $\tilde{\Omega}_{s_{t}}$ in the proof of Proposition 5.2, we see that

$$
\tilde{\Omega}_{t}^{\prime}:=\tilde{\Omega}_{s_{t}}-250 \epsilon \tilde{\Omega}_{0}-\beta^{\prime} \sqrt{-1} \partial \bar{\partial} \ln \left|\pi^{*} \mathcal{S}_{Y}\right|_{h^{N}}^{2}+\beta^{\prime \prime} \sqrt{-1} \partial \bar{\partial} \ln \left|s_{E}\right|_{\otimes h_{i}^{-1}}^{2} \geq \tilde{\Omega}_{s_{t}}+\frac{\tilde{\Omega}_{0}}{K}
$$

for all $\epsilon<\epsilon_{0}$ and all $0<t<1$ away from $\tilde{Y}$. Using Lemma 3.2, we see that $\tilde{\Omega}_{t}^{\prime}$ satisfies the following cone condition pointwise on $\tilde{Z} \backslash(\tilde{Y} \cup \tilde{E})$.

$$
\frac{1}{(1+4 \epsilon)^{2}}\left(\tilde{\Omega}_{t}^{\prime}\right)^{m}-\sum_{j=0}^{m-1} b_{j} \tilde{\chi}_{s_{t}}^{m-j}\left(\tilde{\Omega}_{t}^{\prime}\right)^{j} \geq 0
$$


for some uniform choice of $\epsilon<\epsilon_{0}$ and all $0<t<<1$. Since $\tilde{\chi}_{s_{t}} \leq C_{\chi} \tilde{\Omega}_{0}$ for a uniform $C_{\chi}$ (independent of $t$ ) for all points of $\tilde{M}$, we can apply Lemma 5.3 (and monotonicity in $\Omega$ ) to conclude that there exists $s_{0}^{\prime}$ such that for $s<s_{0}^{\prime}$,

$$
\frac{1}{(1+2 \epsilon)^{2}}\left(\tilde{\Omega}_{t, s}^{\prime \prime}\right)^{m}-\sum_{j=0}^{m-1} b_{j} \tilde{\chi}_{s_{t}, s}^{m-j}\left(\tilde{\Omega}_{t, s}^{\prime \prime}\right)^{j} \geq 0,
$$

where $\tilde{\Omega}_{t, s}^{\prime \prime}=\tilde{\Omega}_{t}^{\prime}(1+10 s)+10 s^{2} \tilde{\Omega}_{0}$ and $\tilde{\chi}_{s_{t}, s}=\tilde{\chi}_{s_{t}}+s^{5 n} \tilde{\Omega}_{0}$.

A weak limit of $\tilde{\Omega}_{t, s}^{\prime \prime}$ is $T_{s}^{\prime}=T_{0}^{\prime}(1+10 s)+10 s^{2} \tilde{\Omega}_{0}$. Hence, its Lelong sublevel set $E_{c}\left(T_{s}^{\prime}\right)$ is contained in $E_{\frac{c}{1+10 s}}(\Theta) \cup \tilde{Y} \cup \tilde{E}$. By shrinking $s_{0}^{\prime}$, we may assume that for all $s<s_{0}^{\prime}, 1+10 s<\frac{3}{2}$ and so $E_{c}\left(T_{s}^{\prime}\right) \subset E_{c / 3}(\Theta) \cup \tilde{Y} \cup \tilde{E}$. By the reasoning in the proof of Theorem 3.1, we see that $T_{s}^{\prime}$ satisfies the desired degenerate cone condition.

Let $c>0$ (independent of $s$ ) be a constant such that $T_{s}$ has a Lelong number $\geq 100 c$ on $\tilde{Y} \cup \tilde{E}$. We now proceed to regularise $T_{s}$ using the regularised maximum construction and glue it with a smooth metric (that we produce using the induction hypothesis) in an appropriately chosen neighbourhood of $\tilde{Y} \cup \tilde{E}$. As before, cover $\tilde{Z}$ with $\tilde{\Omega}_{0}$-balls $B_{2 r}\left(x_{i}\right)$ of radius $2 r<1$ such that $B_{r}^{i}=B_{r}\left(x_{i}\right)$ also cover it and the following hold.

(1) For every $z \in \tilde{Y} \cup \tilde{E}$, there exists an $i$ such that $B_{r / 4}(z) \subset B_{r}^{i}$ where $B_{r / 4}(z)$ is the $\tilde{\Omega}_{0}$-ball of radius $\frac{r}{4}$ around $z$.

(2) On $B_{2 r}^{i}$, there exist holomorphic coordinates such that $\tilde{\Omega}_{0}=\sqrt{-1} \partial \bar{\partial} \phi_{\tilde{\Omega}_{0}}^{i}$, and

$$
\begin{aligned}
& \left.\left|\phi_{\tilde{\Omega}_{0}}^{i}-\right| z\right|^{2} \mid<\epsilon r^{2}, \\
& \quad\left(1-\frac{\epsilon}{200}\right) \sqrt{-1} \partial \bar{\partial}|z|^{2} \leq \tilde{\Omega}_{0} \leq\left(1+\frac{\epsilon}{200}\right) \sqrt{-1} \partial \bar{\partial}|z|^{2} .
\end{aligned}
$$

Define

$$
\epsilon_{5}=\min \left(c, \frac{\epsilon c_{n} r^{2}}{40}\right),
$$

where as before, $c_{n}$ is given by (4.1). Note that this constant is independent of $s$.

The subset $S=\pi\left(E_{\epsilon_{5} / 5}(\Theta) \cup \tilde{Y} \cup \tilde{E}\right) \subset M$ is an analytic subset of maximal dimension $<m$. Hence, by the induction hypothesis, in a neighbourhood $U_{S} \subset M$ of $S$, there exists a constant $0<\epsilon_{1}<<\epsilon$ and a smooth Kähler metric $\Omega_{S}=$ $\Omega_{0}+\sqrt{-1} \partial \bar{\partial} \phi_{S}$ satisfying

$$
\frac{1}{\left(1+4 \epsilon_{1}\right)^{2}} \Omega_{S}^{n-1}-\sum_{k=1}^{n} c_{k} k \chi^{n-k} \Omega_{S}^{k-1}>0 .
$$

Here $\epsilon_{1}<\frac{\epsilon}{3}<1$ is independent of $s$. Note that more precisely, the induction hypothesis, gives the above inequality without the coefficient $\left(1+4 \epsilon_{1}\right)^{-2}$, but since the cone 
condition is pointwise, we can obtain the above inequality, by possibly shrinking $U_{S}$ and compactness. If $s<s_{0}^{\prime}$, then by Proposition 5.3, $S$ contains $\pi\left(E_{\epsilon_{5}\left(T_{s}\right)} \cup \tilde{Y} \cup \tilde{E}\right)$. Since $\pi^{*} \chi \leq C_{\chi} \tilde{\Omega}_{0}$ for a uniform $C_{\chi}$, we see that Lemma 5.3 is applicable to $\Omega=\pi^{*} \Omega_{S}$ and $\chi=\pi^{*} \chi$. Therefore, we get a metric $\Omega_{S, s} \in\left[\pi^{*} \Omega_{S}\right](1+10 s)+10 s^{2}\left[\tilde{\Omega}_{0}\right]$ satisfying

$$
\frac{1}{\left(1+2 \epsilon_{1}\right)^{2}} \Omega_{S, s}^{n-1}-\sum_{k=1}^{n} c_{k} k \chi^{n-k} \Omega_{S, s}^{k-1}>0,
$$

on $\pi^{-1}\left(U_{S}\right)$. Choose $s$ to be (possibly smaller than earlier) that in addition to all the conditions above, the following condition is met.

$$
\left(1+10 s+10 s^{2}\right)^{n}-1<\frac{\epsilon_{1}}{200} .
$$

We do not require any more conditions on $s$ and hence we stick with this choice for the remainder of this section.

Let $\phi_{\Theta}$ be a quasi-plurisubharmonic function on $\tilde{Z}$ such that $\Theta=\tilde{\Omega}_{0}+\sqrt{-1} \partial \bar{\partial} \phi_{\Theta}$. Now denote $\phi_{T_{s}}=\left(\phi_{\Theta}-\beta^{\prime} \ln \left|\pi^{*} \mathcal{S}_{Y}\right|_{h^{N}}^{2}+\beta^{\prime \prime} \ln \left|s_{E}\right|_{\otimes h_{i}}^{2}\right)(1+10 s)$. Define a plurisubharmonic function $\phi_{\delta, s}^{i}$ as the $\delta$-smoothing of $\phi_{T_{s}}+(1-100 \epsilon) \phi_{\tilde{\Omega}_{0}}^{i}$. It is clearly well-defined on $\bar{B}_{9 r / 5}^{i}$.

REMARK 5.2. For all sufficiently small $0<\delta<1$ such that there exists a constant coefficient form $\chi_{0}$ in $B_{\delta}(x)$ satisfying

$$
\chi_{0} \leq \tilde{\chi}_{s} \leq \chi_{0}\left(1+\frac{\epsilon_{1}}{1000000 n^{10 n}}\right),
$$

we see (using the proof of Proposition 5.2 for instance) that the $\phi_{\delta, s}^{i}$ satisfies the strict cone condition

$$
\frac{1}{\left(1+2 \epsilon_{1}\right)^{2}}\left(\sqrt{-1} \partial \bar{\partial} \phi_{\delta, s}^{i}\right)(x)^{m}-\sum_{j=0}^{m-1} b_{j} \tilde{\chi}_{s}^{m-j}\left(\sqrt{-1} \partial \bar{\partial} \phi_{\delta, s}^{i}\right)^{j}(x)>0,
$$

for all $x \in \tilde{Y}^{c} \cap \tilde{E}^{c}$ away from say, $4 \delta$-neighbourhoods (measured using $\tilde{\Omega}_{0}$ ) from $\tilde{Y} \cup \tilde{E}$.

Choose $\delta$ sufficiently small so that for every $x \in \tilde{Z}$, there exists a $\chi_{0}$ as required in Remark 5.2 on $B_{2 \delta}(x)$.

We wish to glue (a small modification) of the metric $\Omega_{S, s}$ with the $\phi_{\delta, s}^{i}$ as before. Choose $\delta$ to be small enough so that on all charts $B_{i}$, the following holds.

$$
\tilde{\Omega}_{s}-\left(\tilde{\Omega}_{s}\right)_{, \delta}+(1-100 \epsilon) \tilde{\Omega}_{0, \delta}-\tilde{\Omega}_{0} \geq-150 \epsilon \tilde{\Omega}_{0, \delta},
$$

Now we need to prove that the "refine" Lelong numbers $\nu(x, \delta)$ of $T$ are somewhat large near $\tilde{Y} \cup \tilde{E}$. Indeed, the singularity of $T$ near $\tilde{E}$ is an explicit logarithmic one. The one near $\tilde{Y}$ is not so explicit. However, Lemma 4.1 implies that for sufficiently 
small $\delta$, the $\delta$-Lelong number $\nu^{i}(x, \delta)$ of $\phi_{T_{s}}+(1-100 \epsilon) \phi_{\tilde{\Omega}_{0}}^{i}$ is larger than $5 \epsilon_{5}$ in a neighbourhood $O_{\delta} \subset \bar{O}_{\delta} \subset \pi^{*} U_{S} \cap \tilde{Z}$ of $\tilde{Y} \cap \tilde{E}$. Choose $\delta$ to be so small that a $10 \delta$-neighbourhood (in the $\tilde{\Omega}_{0}$ sense) of $\tilde{Y} \cup \tilde{E}$ is contained in $O_{\delta}$.

We can now decrease $\delta$ further (if necessary) and define $\psi_{s}$ to be the regularised maximum (at the level $\frac{\epsilon r^{2}}{3}$ ) over the balls $\bar{B}_{9 / 5}^{i}$ of the functions $\phi_{\delta, s}^{i}-\phi_{\tilde{\Omega}_{0}}^{i}$ and $\phi_{S, s}+3.5 \ln \delta$. Using Proposition 4.1 of [CHEN21], we see that $\psi_{s}$ is smooth and coincides with $\phi_{S, s}+3.5 \ln \delta$ in $O_{\delta}$. Moreover,

$$
\begin{aligned}
& \sqrt{-1} \partial \bar{\partial}\left(\phi_{\delta, s}^{i}-\phi_{\tilde{\Omega}_{0}}^{i}\right)+\tilde{\Omega}_{s}=\sqrt{-1} \partial \bar{\partial} \phi_{T_{s}, \delta}^{i} \\
& \quad+\tilde{\Omega}_{s}+(1-100 \epsilon) \sqrt{-1} \partial \bar{\partial} \phi_{\tilde{\Omega}_{0}, \delta}^{i}-\tilde{\Omega}_{0} \\
& \quad=\left(T_{s}\right)_{, \delta}+\tilde{\Omega}_{s}-\left(\tilde{\Omega}_{s}\right)_{, \delta}+(1-100 \epsilon) \tilde{\Omega}_{0, \delta}-\tilde{\Omega}_{0} \\
& \quad \geq\left(T_{s}\right)_{, \delta}-150 \epsilon \tilde{\Omega}_{0, \delta} .
\end{aligned}
$$

By assumption on the smallness of $\delta$, it is a Kähler current throughout $\tilde{Z}$, satisfies the cone condition away from $O_{\delta}$. Hence, $\tilde{\Omega}_{\psi_{s}}=\tilde{\Omega}_{s}+\sqrt{-1} \partial \bar{\partial} \psi_{s}$ satisfies

$$
\frac{1}{\left(1+1.5 \epsilon_{1}\right)^{2}} \tilde{\Omega}_{\psi_{s}}^{m}-\sum_{j=0}^{m-1} b_{j} \tilde{\chi}_{s}^{m-j} \tilde{\Omega}_{\psi_{s}}^{j}>0,
$$

away from $\tilde{E}$ (and in fact, since $\psi$ coincides with $\pi^{*} \phi_{S}$ in $O$, these functions descend to smooth functions on $Z$ and satisfy the cone condition on it).

Now we follow the proof of Lemma 4.4. Extend $\psi$ in the same manner as before to a neighbourhood and consider $\psi_{s}^{\prime}=\psi_{s}+\operatorname{Cdist}(., \tilde{Z})^{2}$ in a small neighbourhood $W_{1}$ of $\tilde{Z}$ in $\tilde{M}$ such that $\tilde{\Omega}_{W_{1}}=\tilde{\Omega}_{s}+\sqrt{-1} \partial \bar{\partial} \psi_{s}^{\prime}$ satisfies the following condition.

$$
\frac{1}{\left(1+\epsilon_{1}\right)^{2}} n \tilde{\Omega}_{W_{1}}^{n-1}-\sum_{k=1}^{n-1} c_{k} k \tilde{\chi}_{s}^{n-k} \tilde{\Omega}_{W_{1}}^{k-1} \geq 0 .
$$

The construction of Lemma 5.3 shows the existence of a metric $\tilde{\Omega}_{W_{2}}$ on a neighbourhood $W_{2}$ of $E$ satisfying the same inequality as in 5.23. As in [CHEN21], we glue (modifications of) these metrics to obtain a metric $\tilde{\Omega}_{U}$ on a neighbourhood of $\tilde{Z} \cup E$ satisfying

$$
\frac{1}{\left(1+0.5 \epsilon_{1}\right)^{2}} n \tilde{\Omega}_{U}^{n-1}-\sum_{k=1}^{n-1} c_{k} k \tilde{\chi}_{s}^{n-k} \tilde{\Omega}_{U}^{k-1} \geq 0 .
$$

On, $W_{1}^{\prime}=\pi\left(\tilde{U} \cap \tilde{E}^{c}\right)$, the function $\psi_{W_{1}^{\prime}, s}^{\prime}=\psi_{s}^{\prime} \circ \pi^{-1}$ is bounded. We define

$$
\psi_{s}^{\prime \prime}:=\frac{\psi_{W_{1}^{\prime}, s}^{\prime}}{1+10 s+10 s^{2}} .
$$

Since $s$ satisfies 5.18, we are done by the arguments of the proof of Lemma 4.4.

Given Proposition 5.1, we can follow the proof of Theorem 1.3 to complete the proof of Theorem 1.1. 


\section{Acknowledgments}

This is work partially supported by grant F.510/25/CAS-II/2018(SAP-I) from UGC (Govt. of India). The first author (V. Datar) is grateful to the Infosys Foundation for their support through the Young Investigator Award. The second author (V. Pingali) is partially supported by a MATRICS grant MTR/2020/000100 from SERB (Govt. of India) We are deeply indebted to Gao Chen for many clarifications regarding his paper, and for his comments on all drafts of this work. We thank Jian Song for his comments on the first draft of the paper, and for many useful discussions on the issue of relaxing uniform positivity in the Theorem 1.3. We also thank Indranil Biswas, Apoorva Khare, Kapil Paranjape, Venkatesh Rajendran for help with regard to some algebraic aspects of the work. Finally, we thank the anonymous reviewer for detailed and useful comments.

Publisher's Note Springer Nature remains neutral with regard to jurisdictional claims in published maps and institutional affiliations.

\section{References}

[BM99] E. Bierstone and P.D. Milman. "Resolution of singularities." Several complex variables (Berkeley, CA, 1995-1996) 37 (1999) : 43-78.

[BK07] Z. Blocki and S. Kolodziej. "On regularization of plurisubharmonic functions on manifolds." Proc. Amer. Math. Soc. 135 (2007): 2089-2093.

[CHEN21] G. Chen. The J-equation and the supercritical deformed Hermitian-YangMills equation. Invent. math. (2021). https://doi.org/10.1007/s00222-02101035-3.

[CHEN00] X. Chen. "On the lower bound of the Mabuchi energy and its application." Int. Math. Res. Notices, 2000(12) (2000): 607-623.

[CLT] J. Chu, M.-C Lee, and R. Takahashi. "A Nakai-Moishezon type criterion for supercritical deformed Hermitian-Yang-Mills equation." arXiv:2105.10725.

[CJY20] T. Collins, A. Jacob, and S.T. Yau "(1, 1) forms with specified Lagrangian phase: A priori estimates and algebraic obstructions." Camb. J. Math, 8(2) (2020): 407-452.

[CS17] T. Collins and G. Székelyhidi "Convergence of the J-flow on toric manifolds." J. Differential. Geom, 107(1) (2017): 47-81.

[CXY18] T. Collins, D. Xie, and S.T. Yau. "The deformed Hermitian-Yang-Mills equation in geometry and physics." Geometry and Physics: Volume 1: A Festschrift in Honour of Nigel Hitchin 1 (2018): 69.

[CY21] T. Collins and S.T. Yau. "Moment Maps, Nonlinear PDE and Stability in Mirror Symmetry, I: Geodesics." Annals of PDE, 7(1) (2021): 1-73.

[DP01] J-P. Demailly and M. Paun. "Numerical characterization of the Kähler cone of a compact Kähler manifold.” Ann. Math, (2004): 1247-1274.

[DK19] R. Dervan and J. Keller. "A finite dimensional approach to Donaldson's J-flow." Comm. Anal. Geom. 27(5) (2019): 1025-1085.

[DON99] S. K. Donaldson. "Moment maps and diffeomorphisms." Asian J. Math. 3 (1999): 1-15. 
[DYR20] Z. Dyrefelt. "Optimal lower bounds for Donaldson's J-functional." Advances in Math. 374(2020): 107271.

[FLM11] H. Fang, M. Lai, and X. Ma. "On a class of fully nonlinear flows in Kähler geometry." J. für die reine ang. Math. 653 (2011): 189-220.

[FLSW14] H. Fang, M. Lai, J. Song, and B. Weinkove. "The J-flow on Kähler surfaces: a boundary case." Anal. PDE, 7(1) (2014): 215-226.

[HJ21] X. Han and X. Jin. "A rigidity theorem for deformed Hermitian-Yang-Mills equation." Calc. Var. Part. Diff. Eq. 60(1) (2021): 1-16.

[HJ] X. Han and X. Jin. "Stability of line bundle mean curvature flow." arXiv:2001.07406 (2020).

[HY] X. Han and H. Yamamoto. "An $\varepsilon$-regularity theorem for line bundle mean curvature flow." arXiv:1904.02391.

[HJ17] Y. Hashimoto and Julien Keller. "About J-flow, J-balanced metrics, uniform J-stability and K-stability." arXiv:1705.02000 (2017).

[HIR64] H. Hironaka. "Resolution of singularities of an algebraic variety over a field of characteristic zero. I" Ann. Math.2, 79(1) (1964): 109-203.

[HIRO64] H. Hironaka. "Resolution of singularities of an algebraic variety over a field of characteristic zero. II" Ann. Math. 2, 79(1) (1964): 205-326.

[JAC] A. Jacob. "Weak Geodesics for the deformed Hermitian-Yang-Mills equation." arXiv:1906.07128.

[JY17] A. Jacob and S. T. Yau. "A special Lagrangian type equation for holomorphic line bundles." Math. Ann. 369(1-2) (2017): 869-898.

[KY20] K. Kawai and H. Yamamoto. "Deformation theory of deformed Hermitian Yang-Mills connections and deformed Donaldson-Thomas connections." arXiv:2004.00532 (2020).

[KOL07] J. Kollár. Lectures on resolution of singularities (AM-166). Annals of Mathematics Studies, Princeton University Press (2007).

[LS15] M. Lejmi and G. Székelyhidi. "The J-flow and stability." Adv. Math. 274 (2015): 404-431.

[PIN19] V. Pingali. "A note on the deformed Hermitian-Yang-Mills PDE." Comp. Var. Ellip. Eq. 64.3 (2019): 503-518.

[PIN14] V. Pingali. "On a generalised Monge-Ampère equation." J. Part. Diff. Eq., 27(4) (2014): 333-346.

[PIN19] V. Pingali. "The deformed Hermitian Yang-Mills equation on three-folds." Analysis and PDE (In press) arXiv:1910.01870 (2019).

[PS09] D. Phong and J. Sturm. "The Dirichlet problem for degenerate complex MongeAmpere equations." arXiv preprint arXiv:0904.1898 (2009).

[RICH68] R. Richberg. "Stetige streng pseudokonvexe Funktionen." Math. Ann. 175 (1968): 257-286.

[SIU86] Y-T Siu. Lectures on Hermitian-Einstein metrics for stable bundles and KählerEinstein metrics: delivered at the German Mathematical Society Seminar in Düsseldorf in June, (1986) (Vol. 8). Birkhäuser.

[SS19] E. Schlitzer and J. Stoppa. "Deformed Hermitian Yang-Mills connections, extended gauge group and scalar curvature." arXiv:1911.10852 (2019).

[SW08] J. Song and B. Weinkove. "On the convergence and singularities of the J-flow with applications to the Mabuchi energy." Comm. Pure Appl. Math. 61(2) (2008): 210-229 
[SW] J. Song and B. Weinkove. "The degenerate J-flow and the Mabuchi energy on minimal surfaces of general type." Univ. Iagellonicae Acta Math. 50 : 89-106.

[SON] J. Song. "Nakai-Moishezon criterions for complex Hessian equations." arXiv:2012.07956.

[SUN] W. Sun. "Generalized complex Monge-Ampère type equations on closed Hermitian manifolds." arXiv:1412.8192.

[SZE18] G. Székelyhidi. "Fully non-linear elliptic equations on compact Hermitian manifolds." J. Differential Geom. 109(2) (2018): 337-378.

[TAK20] R. Takahashi. "Tan-concavity property for Lagrangian phase operators and applications to the tangent Lagrangian phase flow." arXiv:2002.05132 (2020).

[TAK21] R. Takahashi. "Collapsing of the line bundle mean curvature flow on Kähler surfaces." Calc. Var. Part. Diff. Eq. 60(1) (2021): 1-18

[TW10] V. Tosatti and B. Weinkove. "Estimates for the complex Monge-Ampére equation on Hermitian and balanced manifolds." Asian J. Math. 14(1) (2010): 19-40

[TOWE10] V. Tosatti and B. Weinkove. "The complex Monge-Ampére equation on compact Hermitian manifolds." J. Am. Math. Soc. 23(4) (2010): 1187-1195

[WEI04] B. Weinkove. "Convergence of the J-flow on Kähler surfaces." Comm. Anal. Geom. 12(4) (2004): 949-965.

[WEI06] B. Weinkove. "On the J-flow in higher dimensions and the lower boundedness of the Mabuchi energy." J. Differential Geom. 73(2) (2006): 351-358

[XIA16] J. Xiao. "Positivity in convergence of the inverse $n$-1-flow." arXiv:1610.09584 (2016).

[XIAO16] J. Xiao. "A remark on the convergence of the inverse $\sigma$-flow." Comp. Rend. Math. 354(4) (2016): 395-399.

[YAM18] H. Yamamoto. "Special Lagrangian and deformed Hermitian Yang-Mills on tropical manifolds." Math. Zeit. 290(3-4) (2018): 1023-1040

[YAO15] Y. Yao. "The J-flow on toric manifolds." Acta Math. Sinica, English Series, 31(10) (2015): 1582-1592

[YAU78] S.-T. Yau. "On the Ricci curvature of a compact Kähler manifold and the complex Monge-Ampère equation, I." Comm. Pure Appl. Math. 31(3) (1978): $339-411$.

[ZHE15] K. Zheng. "I-properness of Mabuchi's K-energy." Calc. Variations Par. Diff. Eq, 54(3) (2015): 2807-2830.

Ved V. Datar • VAmsi Pritham Pingali

Department of Mathematics, Indian Institute of Science, Bangalore 560012, India.

vvdatar@iisc.ac.in; vamsipingali@iisc.ac.in

Received: May 11, 2021

Revised: July 29, 2021

Accepted: August 2, 2021 